\title{
FluidFM for single-cell biophysics
}

\author{
Mi Li ${ }^{1,2,3}(\bowtie)$, Lianqing $\mathrm{Liu}^{2,3}$, and Tomaso Zambelli ${ }^{1}$ \\ ${ }^{1}$ Laboratory of Biosensors and Bioelectronics, Institute for Biomedical Engineering, ETH Zürich, Zürich 8092, Switzerland \\ ${ }^{2}$ State Key Laboratory of Robotics, Shenyang Institute of Automation, Chinese Academy of Sciences, Shenyang 110016, China \\ ${ }^{3}$ Institutes for Robotics and Intelligent Manufacturing, Chinese Academy of Sciences, Shenyang 110169, China
}

(C) The Author(s) 2021

Received: 4 January 2021 / Revised: 16 April 2021 / Accepted: 5 May 2021

\begin{abstract}
Fluidic force microscopy (FluidFM), which combines atomic force microscopy (AFM) with microchanneled cantilevers connected to a pressure controller, is a technique allowing the realization of force-sensitive nanopipette under aqueous conditions. FluidFM has unique advantages in simultaneous three-dimensional manipulations and mechanical measurements of biological specimens at the micro-/nanoscale. Over the past decade, FluidFM has shown its potential in biophysical assays particularly in the investigations at single-cell level, offering novel possibilities for discovering the underlying mechanisms guiding life activities. Here, we review the utilization of FluidFM to address biomechanical and biophysical issues in the life sciences. Firstly, the fundamentals of FluidFM are represented. Subsequently, the applications of FluidFM for biophysics at single-cell level are surveyed from several facets, including single-cell manipulations, single-cell force spectroscopy, and single-cell electrophysiology. Finally, the challenges and perspectives for future progressions are provided.
\end{abstract}

\section{KEYWORDS}

atomic force microscopy, fluidic force microscopy, single-cell manipulation, single-cell force spectroscopy, single-cell electrophysiology

\section{Introduction}

It is increasingly evident that investigating the physiological/ pathological processes at single-cell level is of remarkable significance for unveiling the mysteries of life activities. Traditional biological assays are based on ensemble measurements performed on cell populations, which are undoubtedly powerful tools for the identification of components and interactions within complex metabolic, signaling, and transcriptional networks [1]. Nevertheless, it should be noted that cell-to-cell variability is not apparent in population-averaged assays [2]. It is widely known that single cells in a population display variable behaviors [3] and even genetically identical cells grown in homogeneous environments exhibit different phenotypes [4]. The stochasticity in molecular processes such as stochastic gene expression [5] has been found to be related to the cell-to-cell phenotypic variability. Particularly, for tumors, intratumor (within a tumor, subclonal diversity exists) and intercellular (within a subclonal population of tumor cells, tumor cells exhibit different behaviors) heterogeneity have been widely observed [6,7], which are key factors that may lead to drug resistance and pose challenges for the design of clinical trials [8]. Hence, investigating life activities at single-cell level significantly benefits the reveal of the novel underpinnings regulating physiological/pathological processes, which has been inaccessible for a long time due to the lack of study tools. In the past years, various single-cell biochemical methods have emerged to profile genetic, epigenetic, spatial, proteomic and lineage information in individual cells [9], including single-cell ribonucleic acid (RNA) sequencing [10], single-cell deoxyribonucleic acid (DNA) methylation profiling [11], single-cell western blotting [12], and so on. With the use of these single-cell biochemical assays, unforeseen processes and patterns in life sciences have been revealed [13], such as intratumoral heterogeneity in primary glioblastoma [14], the random monoallelic gene expression in mammalian cells [15], and the identification of somatic mutations in single cell [16].

Investigating single-cell mechanics has emerged as a promising way for understanding the biological processes. Life is not only a chemical process, but also a mechanical one [17]. In fact, mechanical forces are increasingly recognized as major regulators of cell structure and function. The mechanical properties of cells are essential to the mechanisms by which cells sense external mechanical cues and transduce them into chemical signals for regulating various biological processes at the molecular and cellular level [18], such as gene expression, adhesion, migration, and cell fate [19]. Abnormal alterations in cell mechanics often influence, and are influenced by, the onset and progression of human diseases such as cancer [20]. For example, it has been found that in addition to genetic and external environmental factors, the physical interactions of cancer cells with their microenvironment, as well as their modulation by mechanical forces, are key determinants of the metastatic process of tumor [21]. Therefore, studying cell mechanics is meaningful for identifying the physical biomarkers in physiological/pathological processes [22], which significantly complements traditional biochemical assays for thoroughly understanding life activities. Particularly, single-cell biochemical assays require various pretreatments of cells, such as lysis, fixation, staining, and labeling [14-16, 23], meaning that cells 
lose biological activities after these treatments. On the contrary, single-cell mechanical measurements are directly performed on living cells and the biological functions (such as invasion and metastatic capabilities [24]) of the same cells can further be examined by using biochemical assays, which facilitates investigating the direct association between cell mechanics and cell functions at the single-cell level and therefore is of fundamental significance for revealing the heterogeneous behaviors of single cells.

The advent of fluidic force microscopy (FluidFM) [25] provides a powerful multifunctional toolbox for the studies of mechanics at the single-cell level. FluidFM is derived from atomic force microscopy (AFM) [26]. AFM uses a sharp tip mounted at the end of a microcantilever to probe the specimens immobilized on the support, and the unique advantage of AFM is that it is able to work under aqueous conditions with nanometer spatial resolution and millisecond temporal resolution [27], making it particularly suited for the studies of living biological specimens in their native state. Besides resolving the topography at diverse imaging modes [28], AFM has achieved unprecedented success in detecting the mechanical properties of biological specimens in force spectroscopy mode [29], significantly contributing to the field of mechanobiology. In addition to AFM, various emerging techniques are available for measuring the mechanics of single cells, including microfluidics, micropipette aspiration, micropost arrays, magnetic twisting cytometry (MTC), optical stretching, parallel-plate rheometry, particle-tracking microrheology (PTM), and cell monolayer rheology (CMR) [30-34], each of them with its advantages and limitations [30-34]. In practice, AFM is the most widely used tool for single-cell mechanical analysis [35-38]. However, regular AFM probe can only perform simple mechanical motions on cells in the horizontal plane, such as touching, compressing, indenting, pushing, stretching and cutting [39]. By mounting a nanoneedle [40] or nanotube [41] probe onto the AFM head, molecules could be delivered into single cells, but such protocol required the covalent conjugation of target molecules onto the surface of AFM probe and was dependent on reversible chemical reactions, which decreased the fidelity of target molecules and caused limited controllability. Inspired by the glass micropipette [42] which is a widely used tool for cell manipulation, FluidFM takes advantage of microchanneled
AFM cantilevers connected to a pressure controller [43]. In this way, FluidFM integrates the advantages of micropipette and AFM, since FluidFM can not only perform three-dimensional manipulations of single cells, but also tune liquid delivery to single cells with high precision (femtoliter) and perform mechanical measurements on single cells with nanometer spatial resolution, providing new possibilities for the studies at single-cell level. Recently, Saha et al. [44] have surveyed the applications of FluidFM on force measurements. Here, the advances of FluidFM in single-cell biophysics (including singlecell manipulations, single-cell force spectroscopy (SCFS), and single-cell electrophysiology) are comprehensively summarized and illustrated with examples highlighting the unique capabilities of FluidFM. The challenges and future progressions are also discussed.

\section{Fundamentals of FluidFM}

The principle of FluidFM is shown in Fig. 1. FluidFM is based on AFM. An AFM is commonly composed of a piezoelectric tube driver which is connected to a probe, a laser generator, a four-quadrant position sensitive detector (PSD), a signal processing module and the feedback control electronics [45]. AFM probe raster scans the surface of the specimen, and a laser beam is reflected from the backside of the cantilever to the PSD to detect the cantilever deflection. During $X-Y$ scanning, changes in PSD signals are analyzed by the signal processing and input into the feedback module which controls the $Z$ (vertical) movement of the piezoelectric tube driver in order to maintain the feedback parameters constant (e.g., cantilever deflection and amplitude of the vibrating cantilever), thus yielding a three-dimensional topography of the specimen. The basic components of FluidFM are the same as AFM, except that FluidFM uses a microchanneled cantilever which is connected to a pressure controller by tubing, as shown in Fig. 1(a). FluidFM thus has a continuous and closed fluidic channel that can be filled with an arbitrary chosen liquid, which can be locally dispensed through the nanoscale aperture at the extremity of the cantilever [25]. The design of FluidFM gives AFM exciting novel capabilities; for example, it allows AFM to be able to easily perform precise threedimensional manipulations on individual living cells (e.g.,
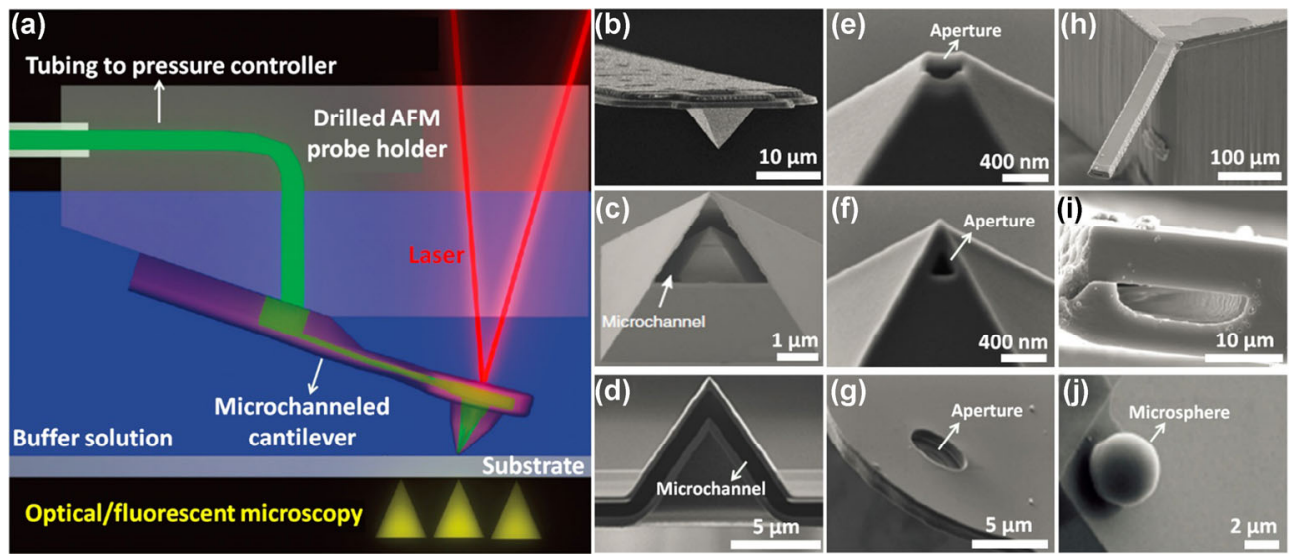

Figure 1 FluidFM configuration. (a) Schematic of FluidFM. The microchanneled cantilever is fixed to the drilled AFM probe holder, which is immersed in liquid on top of an inverted optical/fluorescent microscope. (Reprinted with permission from Ref. [25], ๑ American Chemical Society 2009). (b)-(j) SEM images of the different types of microchanneled probes. (b) The whole probe. (c) Cutaway view of the pyramidal tip with embedded microchannel. (d) Hollow pyramid after sectioning the probe with FIB. (e) and (f) Aperture at the apex (e)/side (f) of the pyramidal AFM tip. (g) Aperture formed in tipless cantilever. (h) Whole image and (i) magnified image of the hollow probe fabricated by SU-8. (j) Microsphere tip prepared based on the microchanneld cantilever. (b) and (d)-(g) are reprinted with permission from Ref. [48], ๑ Elsevier Ltd. 2014. (c) is reprinted with permission from Ref. [49], ( ) Springer Nature 2019. (h) and (i) are reprinted with permission from Ref. [50], @ IOP Publishing Ltd. 2016. (j) is reprinted with permission from Ref. [51], ( ) The Royal Society of Chemistry 2017. 
adsorbing, extracting, transporting, placing, delivering, and injecting) [46] by controlling the pressure of the fluidic channel in the AFM cantilever. Besides, experiments have shown that even though the hollow cantilever is influenced by small changes in pressure, the overall system can suppress this effect in order to ensure constant interaction forces with the specimen at all times during an experiment [43], making the FluidFM probe a highly force-sensitive nanopipette.

Diverse FluidFM probes have been developed for biomedical applications. The FluidFM cantilever is rectangular shaped ( $\sim 150 \mu \mathrm{m}$ long and $25 \mu \mathrm{m}$ long) with an embedded $1-\mu \mathrm{m}$ high microfluidic channel supported by parallel rows of pillars and an aperture at the free extremity. First, hollow cantilevers are produced on a wafer scale combining photolithography for standard AFM probe fabrication and additional protocols based on the "sacrificial layer" principle for fluidic functionality $[46,47]$ After that, an aperture on the AFM tip can be formed either by photolithography (parallel process) or by focused ion beam (FIB) (serial process) [25]. FluidFM probes are commercially available (Cytosurge AG, Switzerland), facilitating the FluidFM studies. With these methods, different types of microchanneled probes can be fabricated [48, 49], as shown in Figs. 1(b)-1(g). From the scanning electron microscopy (SEM) images of the fabricated hollow cantilevers, the microchannels in the cantilever are distinctly discernible (Figs. 1(c) and 1(d)). The aperture can be formed at the apex of the tip (Fig. 1(e)), at the side of the tip (Fig. 1(f)), or directly in the tipless cantilever (Fig. 1(g)) for different experimental purposes. Besides, polymer-based hollow probes (Figs. 1(h) and 1(i)) can be fabricated by using the epoxy-based photoresist SU-8 [50], offering a new alternative to conventional silicon-based hollow cantilevers with more flexibility in terms of complex geometric design and surface chemistry modification. Particularly, based on FluidFM, AFM tips with special shapes can be prepared in a direct way; for example, attracting single microspheres to the aperture of the probe by applying underpressure yields the microsphere probe [51] (Fig. 1(j)). A notable point is that the hollow probes need to be functionalized with an antifouling coating prior to experimentation to avoid biofouling (biofouling is the nonspecific adsorption of biomacromolecules to the probe surface, which can cause the clogging of the flow through the channel in the probe) [52]. The hydrophilic copolymer poly(L-lysine)graft-poly(ethylene glycol) (PLL-g-PEG), the gold standard for nonfouling surface coating [53], has been extensively used to confer antifouling properties of the FluidFM probes [52]. Recently, researchers have developed a simple protocol for the antifouling modifications of FluidFM probes, relying on the protein resistant copolymer called PAcrAm-g-(PMOXA, NH2, $\mathrm{Si})[49,54,55]$. For this method, FluidFM probes are first treated by oxygen plasma and then immersed in a PAcrAmg-(PMOXA, NH2, Si) solution for $90 \mathrm{~min}$ at room temperature. The FluidFM probes can then be used for experiments after rinsing with pure water.

\section{FluidFM for single-cell manipulations}

\subsection{Picking and placing single cells}

By approaching the FluidFM probe onto a cell in contact mode and applying a underpressure in the fluidic channel [48], the cell can be tightly attracted to the aperture of the cantilever ((I) in Fig. 2(a)) in the vertical direction and therefore can be picked up detaching from the surface. By reversing the pressure, the cell can be released onto a desired spot ((II) in Fig. 2(a)). For adherent cells which can attach to and spread on the substrate, the prerequisite of picking up the cell is removing

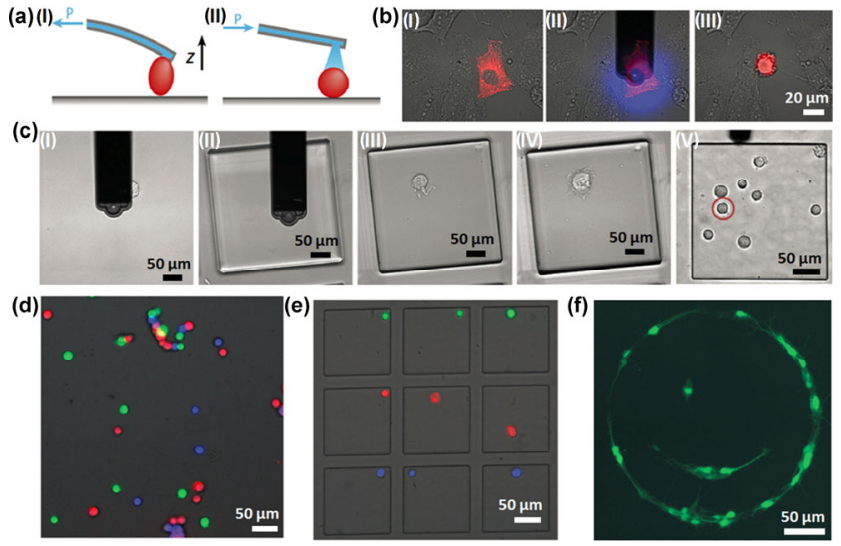

Figure 2 Manipulating individual cells with FluidFM. (a) Schematic of adsorbing (I) or releasing (II) single cells by controlling the pressure of the fluidic channel in the probe. (Reprinted with permission from Ref. [48], (C) Elsevier Ltd. 2014). (b) Single-cell detachment by controlled trypsin release. (I) HeLa cells were cultured on glass and transfected with fluorescence. (II) The microfluidic probe was positioned above the cell to release trypsin. (III) The targeted cell detached. (c) Serial transferring of cells in one microwell. (d) Cells labeled with different fluorescent dyes. (e) After FluidFM manipulations, cells were sorted according to the fluorescence. (b)-(e) are reprinted with permission from Ref. [56], (C) The Royal Society of Chemistry 2014. (f) Smiley pattern formed by cells with the assistance of FluidFM manipulations. (Reprinted with permission from Ref. [57], (C) The Royal Society of Chemistry 2016).

the molecular anchors between cell and substrate. Taking advantage of the FluidFM multifunctionality, trypsin solution can be loaded in the channel and locally delivered to individual adherent cells [56] ((II) in Fig. 2(b)), which activates the selective detachment (rounding) of the targeted cell while neighboring cells remain adherent ((III) in Fig. 2(b)). The detached cell can then be easily lifted up and manipulated by the FluidFM, providing an efficient way for precise isolation of chosen cells in a cell population. In this way, we can transfer cells to targeted areas to observe cell behaviors (Fig. 2(c)) and we can also sort different types of cells under the guidance of fluorescent labeling (Figs. 2(d) and 2(e)). Besides, FluidFM is able to assist in the formation of cell patterns [57] (Fig. 2(f)). Patterning of cells is a critical physiological process for regulating the life activities such as embryonic development and tissue morphogenesis $[58,59]$ and investigating cell patterning behaviors benefits the understanding of the physiological/pathological processes. With FluidFM-based single-cell manipulations, we can not only place cells at specific positions on the same substrate or even of another substrate, but also can remove unwanted cells to facilitate forming desired cell patterns [57], which is useful for the studies of patterning at single-cell level.

\subsection{Sucking colloidal beads as indenters}

FluidFM opens the way to exchangeable colloidal AFM for singlecell mechanical assays. To probe the mechanical properties of cells, spherical AFM tips $[60,61]$ are commonly used, since the conventional conical tips only detect the local mechanics of the cell. Spherical tips have well-defined geometry, which facilitates theoretical modeling of the indenting process [62]. Besides, spherical tips prevent the local damage of the cell membrane caused by the serial poking of AFM sharp tips during indentation assays, for thus more reliable and safer measurements are obtained using spherical tips [63]. In the case of FluidFM, a single sphere can easily be aspirated to the aperture by applying a negative pressure [64]. Both tipless ((I) in Fig. 3(a)) or pyramidal FluidFM probes ((II) in Fig. 3(a)) can be used to prepare spherical tip, and specifically pyramidal probes are suited for preparing spherical tips with smaller 

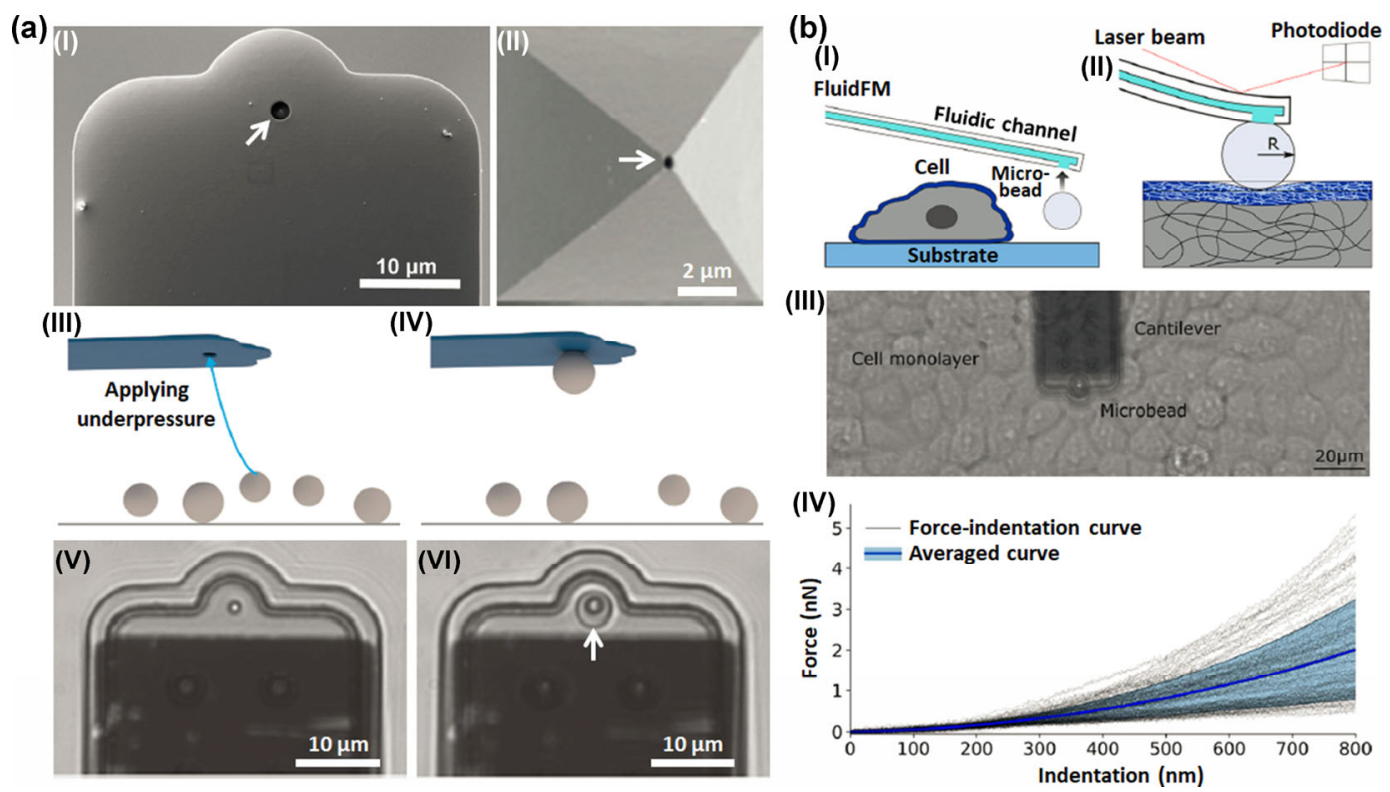

Figure 3 Preparing spherical tip for indenting single cells by FluidFM. (a) Process of preparing spherical tip. (I) and (II) SEM images of tipless FluidFM probe (I) and pyramidal FluidFM probe (II) respectively. The apertures of the probe are clearly discernible, as denoted by the arrows. (III) and (IV) Schematic of applying underpressure to suck single sphere (III) and the fabricated sphere tip (IV). (V) and (VI) Optical images of the FluidFM before (V) and after (VI) sucking a sphere (denoted by the arrow). (I) is reprinted with permission from Ref. [64], ๑ Biophysical Society 2013. (II)-(VI) are reprinted with permission from Ref. [51], @ The Royal Society of Chemistry 2017. (b) Using the FluidFM-prepared spherical tip to indent single cells. (I) and (II) Schematic of picking a sphere with FluidFM to indent the cell on the substrate (I) and sense the mechanics of the cell (II), respectively. (III) Optical image showing probing cells with the spherical tip. (IV) Typical force-indentation curves obtained on the cells. (Reprinted with permission from Ref. [65], (c) Springer Nature 2020).

sizes. Under the guidance of optical microscopy, the FluidFM probe is moved to touch individual spheres deposited on the substrate ((III) and (V) in Fig. 3(a)). Subsequently, applying adequate underpressure to the fluidic channel of the probe allows the adsorption of the sphere to the aperture of the probe ((IV) and (VI) in Fig. 3(a)) [51]. The spherical probes prepared by FluidFM can then be used for experiments such as probing the mechanics of single cells (Fig. 3(b)). Experimental results have significantly shown the capabilities of the FluidFM-prepared spherical tips in sensing the mechanical properties of cells [65]. A notable point is that the sphere sucked to the FluidFM cantilever can be exchanged in the working medium when probing cells. Applying positive pressure yields the release of the sucked sphere, and then moving the probe to the region of the substrate where the colloidal beads had been previously deposited and applying negative pressure will adsorb a new clean one. This is particularly useful for probing the specific molecular interactions which require the functionalization of the tip (e.g., linking specific molecules to the surface of the tip [66]). For example, we can firstly approach a cell with a bare sphere as control, and then detect specific molecular interactions on the cell surface with an ad-hoc chemically functionalized sphere.

\subsection{Dispensing biomolecules on top of single cells}

FluidFM is able to deliver not only trypsin, but whatever biomolecules as chemical stimuli to selected living cells on a Petri dish. Under the guidance of optical microscopy, the microchanneled probe filled with a particular chemical solution is gently approached onto a selected cell relying on the AFM feedback and maintained in contact with the cell membrane ((I) in Fig. 4(a)). Subsequently, by applying adequate overpressure, the solution inside the channel can be released to the cell under the probe. Taking a fluorescent dye solution as in [25], which is known to diffuse into the cytoplasm of the targeted cell, the obtained fluorescent images ((II) and (III) in Fig. 4(a)) clearly confirm the successful staining of the targeted cell. In
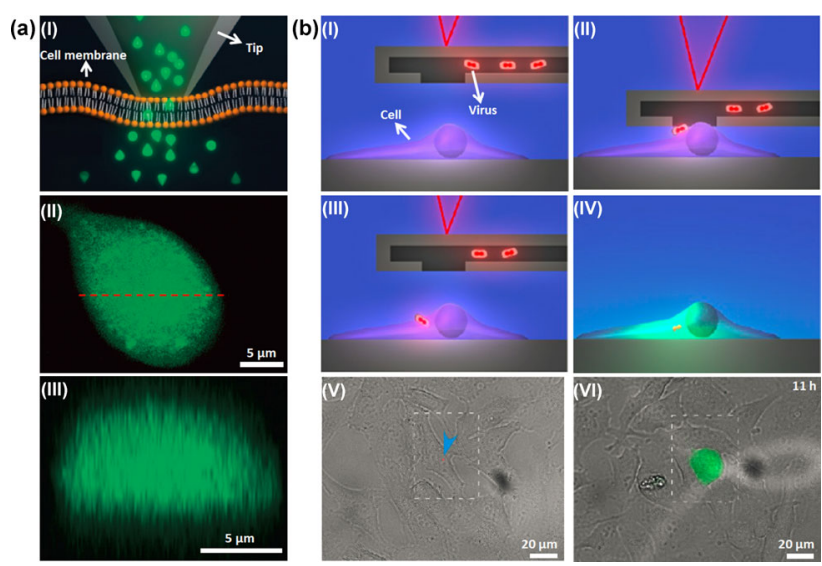

Figure 4 Delivering chemical stimuli to single cells by FluidFM dispensing. (a) Locally delivering fluorescent dye molecules to single cells by diffusion. (I) Schematic. (II) Confocal fluorescence image of the cell after fluorescein isothiocyanate (FITC) dispensing. (III) Confocal cross section image along the dashed red line in (II). (Reprinted with permission from Ref. [25], (C) American Chemical Society 2009). (b) Delivering a single virus to a single living cell. (I)-(IV) Schematics showing the procedure of delivering single virus on the targeted cell. (V) Superposition of the differential interference contrast images and the corresponding fluorescence images of the virus (labeled with red fluorescence) deposited on the cell (V). (VI) Expression of green fluorescent protein detected on cells $11 \mathrm{~h}$ after viral infection. (Reprinted with permission from Ref. [67], (c) American Chemical Society 2012).

addition to small molecules, single virus can be delivered to the targeted cell by FluidFM dispensing [67]. The microchannel filled with the solution containing a virus conjugated with fluorescein for visual recognition, is brought into gentle contact with an optically selected cell ((I) and (II) in Fig. 4(b)): Applying an adequate pressure results in the release of a single virus to the targeted cell ((III) in Fig. 4(b)). The viral infection process on the targeted cell can then be monitored by fluorescence microscopy ((V) and (VI) in Fig. 4(b)). These 
experimental results $[25,67]$ show that liquids can be locally delivered to single cells with volume control by the FluidFM, which is particularly promising for investigating the singlecell behavior in response to external chemical stimuli such as drugs.

\subsection{Intracellular injection of single cells}

Using a pyramidal tip with a side-hole preserving the sharpness of the apex, FluidFM is able to puncture the cell membrane for intracellular delivery. Membrane perforation ((I) in Fig. 5(a)) is indeed achieved by exerting a higher indentation force on the cell surface, which is visually confirmed by the abrupt peak in the recorded force curves [25]. Subsequently, by applying overpressure to the fluidic channel of the probe, the solution inside the microchannel can be directly injected to the interior of the cell. For example, experiments have clearly shown that the injected dye molecules (that cannot otherwise diffuse across the cell membrane) successfully stain the cytoplasm of the targeted cell ((II) in Fig. 5(a)). Besides, FluidFM can even directly deliver liquids into the nucleus of single cells [68]. Typical events during the piercing process can be determined from the force curves ((I) in Fig. 5(b)), including penetration of upper cell membrane, penetration at the top of the nuclear envelope, penetration at the bottom of the nucleus envelope, penetration of lower cell membrane, also confirmed by confocal fluorescent imaging [69] ((II) in Fig. 5(b)). With this method, plasmid DNAs encoding green fluorescent protein (GFP) have been injected into the cell nucleus, and two days after injection the cell successfully produces GFP confirmed by fluorescence (Fig. 5(c)) [68]. Notably, delivering biomolecules via membrane perforation (Fig. 5) is destructive, but this way allows fast delivery to targeted cells and thus facilitates improving efficiency. On the contrast, delivering biomolecules via dispensing (Fig. 4) is non-destructive to cells, but this way is dependent on the diffusion of the dispensed biomolecules, which is much slower than membrane perforation. Consequently, these two methods of biomolecules delivery (dispensing and intracellular injection) provided by FluidFM are complementary and can be used flexibly in practice for different experimental purposes.

\subsection{Intracellular extraction of single cells}

FluidFM allows quantitatively extraction of the intracellular content of single living cells with spatiotemporal control for downstream analyses [70]. The procedure of intracellular structure extraction on single living cells is schematically shown in Fig. 6(a). The procedure consists of controlling the tip of the microchanneled probe to insert into the targeted cell and then applying adequate negative pressure to extract intracellular structures, followed by withdrawing the probe from the cell and dispensing the extraction on the substrate for analyses.

With this generic method, we can not only extract from the nucleus of single living cell (Fig. 6(b)), but also from the cytoplasm on the living cell (Fig. 6(c)). Cells expressing GFP were used to facilitate visually observing the extraction process (Figs. 6(b) and 6(c)) and the extracted intracellular structures (Fig. 6(d)). Since the geometrical parameters of the hollow probe are known, the volumes of the extracted structures can then be precisely quantified according to the fluorescent image of the extraction (Fig. 6(d)). The subsequent cell viability assays showed that after the extraction of volumes up to $4.0 \mathrm{pL}$ from the cytoplasm, $82 \%$ of the cells remained viable, indicating that the cells have the ability to withstand the loss of a large proportion of the cytoplasm. The cells were still viable after the extraction of volumes up to $0.6 \mathrm{pL}$ from the nucleus, indicating that extraction from the cell nucleus was more critical than extraction from the cytoplasm. The extracted intracellular structures of the targeted cell can then be used for various biochemical analyses, including structure characterizations, gene expression analysis, enzyme activity assay, mass spectroscopy, and so on [70,71]. We know that there are not only biochemical alterations but also mechanical changes
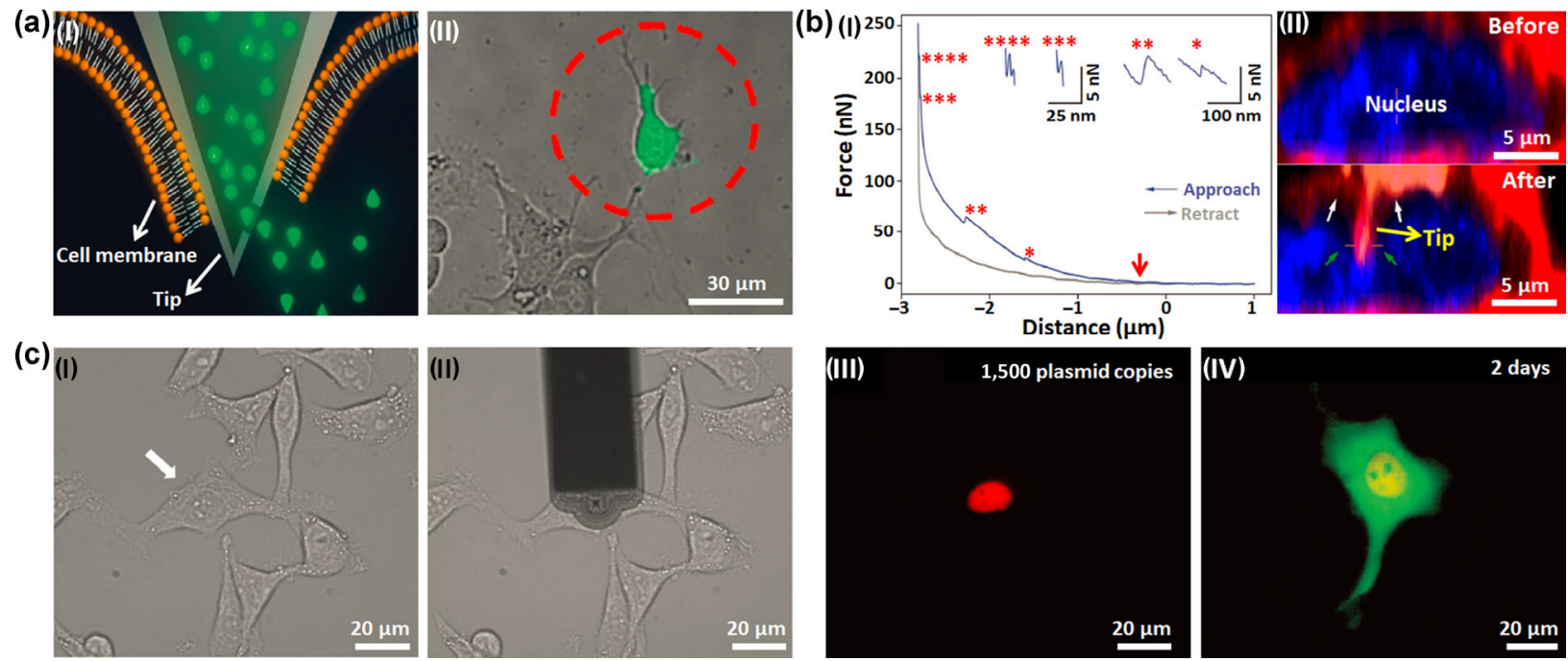

Figure 5 Delivering biomolecules into single cells by FluidFM-based membrane perforation. (a) Injecting fluorescent dye molecules into single cells. (I) Schematic. (II) Superposition of the differential interference contrast image and the corresponding fluorescence image of the cell (denoted by the dashed red circle) after injection with fluorescent dyes. (Reprinted with permission from Ref. [25], () American Chemical Society 2009). (b) Forcecontrolled intranuclear injection. (I) A typical force curve recorded during the process of delivering biomolecules to cell nucleus. Cell indentation started upon contact of the probe with the cell (denoted by the red arrow). During the penetration, the probe passed through several cell structures, which resulted in the corresponding abrupt peaks in the force curve, including upper cell membrane $\left({ }^{*}\right)$, upper nuclear envelope $\left({ }^{* *}\right)$, lower nucleus $\left({ }^{* * *}\right)$, and lower cell membrane ${ }^{(* * *)}$. The insets are the magnified curves of abrupt peaks. (Reprinted with permission from Ref. [68], ( $\odot$ Wiley-VCH Verlag GmbH \& Co. KGaA 2013). (II) Confocal fluorescence images showing that the conventional AFM probe reached cell nucleus (blue) after penetration. (Reprinted with permission from Ref. [69], ( $\odot$ American Chemical Society 2014). (c) Results of delivering plasmid DNAs to cell nucleus. (I) and (II) Phase contrast images of the cell (denoted by the white arrow) before (I) and during (II) injection. (III) and (IV) Fluorescence images of the cell immediately (III) and two days (IV) after injection. (Reprinted with permission from Ref. [68], @ Wiley-VCH Verlag GmbH \& Co. KGaA 2013). 


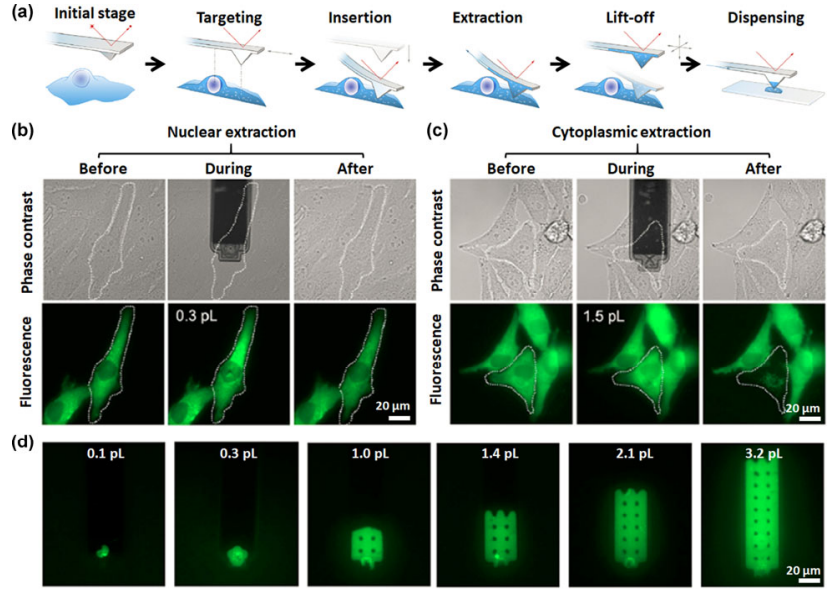

Figure 6 Extracting the intracellular structures of single living cells by FluidFM for molecular analyses. (a) Schematic of the FluidFM-based single living cell extraction procedure. The microchanneled cantilever is monitored by the laser (red) and allows for handling femtoliter liquid volumes with nanometer spatial precision and piconewton force resolution. (b) and (c) Phase contrast and fluorescent images of nuclear extraction (b) and cytoplasmic extraction (c). In the examples shown, $0.3 \mathrm{pL}$ of the nucleoplasm content was extracted (b) and $1.5 \mathrm{pL}$ of the cell cytoplasm was extracted, respectively. (d) Different volumes of GFP-containing extracts collected in the hollow probes. (Reprinted with permission from Ref. [70], @ Elsevier Inc. 2016).

in physiological/pathological process of cells [72], and a major challenge facing cell biology is understanding how the chemical and physical properties come together to guide cell processes [73]. With FluidFM, we can probe the various mechanical properties of single living cells [32], after which the intracellular structures of the same cell can be quantitatively extracted for biochemical assays [70,71], therefore providing a feasible way for directly investigating the relationship between cellular mechanics and cellular chemical properties at single-cell level, which is considerably significant for comprehensively understanding cellular processes.

\subsection{FluidFM limitations for cellular manipulations}

The serial interplay of underpressure to suck a cell against the aperture and of overpressure to release it is sound and uncomplicated. However, an inescapable point is the deposition of an antifouling coating to minimize the possible cell binding at the aperture. This becomes particularly critical when the aspirated cell has to be released onto another substrate, which is favored when the interaction with the new substrate is bigger than that with the aperture. Injection and extraction protocols being linked with membrane perforation are subject to an intrinsic variability because they finally depend on the sharpness of the particular probe being utilized at that particular moment. Moreover, an antifouling coating on the walls of the microchannel is critical also for extraction to avoid unwanted adsorption of the extracted molecules on the walls, and thus being no more available for the planned further analysis.

\section{FluidFM for single-cell force spectroscopy}

\subsection{Adhesion of eukaryotic cells}

The adhesion forces between single living cells and substrates can be quantified by FluidFM. Traditional AFM-based SCFS [74] is based on attaching a living cell to the AFM cantilever via complex chemical functionalization (for example, according to the established protocol in SCFS [75], the cantilever is firstly coated by biotin-labeled bovine serum albumin, and then streptavidin is coated on the cantilever, which is followed by the coating of biotin-labeled concanavalin A), which is laborintensive and requires specific expertise [76]. Particularly, chemically immobilizing the cell onto the AFM cantilever may inevitably influence the activities of the cell (e.g., cell surface damage and denaturation [77]). With the use of FluidFM, single living cells can easily be physically immobilized onto the AFM cantilever by applying and maintaining adequate negative pressure of the fluidic channel in the AFM probe, allowing the SCFS measurements in native conditions (without chemical treatment on cells). Besides, regular functionalized probes can be used only once, whilst cells are prone to detach from the cantilever during SCFS experiments while the FluidFM probe can be reused (after completing the measurements of one cell, the FluidFM probe can be controlled to adsorb another cell for further measurements) and the adsorption force for immobilizing cell can be conveniently adjusted, therefore FluidFM facilitates increasing the throughput and efficiency of SCFS assays [78].

Based on FluidFM, in 2012, Potthoff et al. [79] firstly showed the serial SCFS measurements using a single cantilever for sensing the adhesive interactions of multiple mammalian cells (HeLa cells and HEK cells). Besides, the adhesion forces of human umbilical vein endothelial cells (HUVECs) on different surface topographies were measured by FluidFM [80], benefitting the reveal of the effects of surface textures on promoting endothelialization. In 2016, McGrath et al. [81] showed the capabilities of FluidFM-based SCFS in assessing the deformable properties of oocyst cells. In 2017, Sankaran et al. [82] investigated the adhesion interactions between single cells (mouse myoblast $\mathrm{C} 2 \mathrm{C} 12$ cells) and different types of substrates ((I) in Fig. 7(a)). Two types of RGD-presenting surfaces (non-covalent surface and covalent surface) were analyzed. After adsorbing single living $\mathrm{C} 2 \mathrm{C} 12$ cell with FluidFM probe, the cell probe was controlled to perform approach-retract movement in the vertical direction on the substrates (non-covalent surface or covalent surface), during which force curves ((II) in Fig. 7(a)) were recorded. From the force curve, several parameters reflecting the unbinding between cell and substrate can be obtained, including adhesion force ((III) in Fig. 7(a)), detachment distance ((IV) in Fig. 7(a)), and the binding energy ((V) in Fig. 7(a)), facilitating quantitatively understanding the adhesive interactions between cells and substrates. FluidFM-based SCFS has also been utilized to address various issues regarding cell adhesion, including the effect of electric current on cell adhesion [83], intercellular adhesion forces in mature cell-cell contacts [84], the adhesion of cancer cells at various stages of malignant transformation [85], cell-sheet engineering [86], cell-material interaction [87], and single-cell adhesion assays with high throughput [88], contributing much to the studies of single-cell adhesion in physiological/pathological processes.

\subsection{Adhesion of microbial cells}

FluidFM-based SCFS benefits the exploring of the underlying mechanisms guiding the adhesion interactions of individual microbial cells. Biofilms, a complex group of microbial cells that adhere to the exopolysaccharide matrix present on the surface of medical devices, play an important role in the pathogenesis of implant infections [89]. Originally microbes attach onto the surface of biomaterials, after which the intercellular interactions mediated by adhesins and cell wall proteins lead microbes to cluster together, forming microcolonies and biofilms [90]. Cell adhesion is mediated by a multitude of molecular interactions that are specific (i.e., molecular recognition between receptors and ligands) or non-specific (i.e., hydrogel bonding, hydrophobic, van der Waals, electrostatic, 
(a) (I)

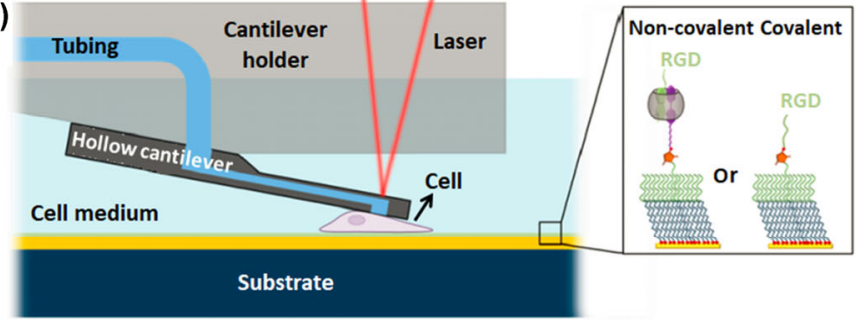

(II)
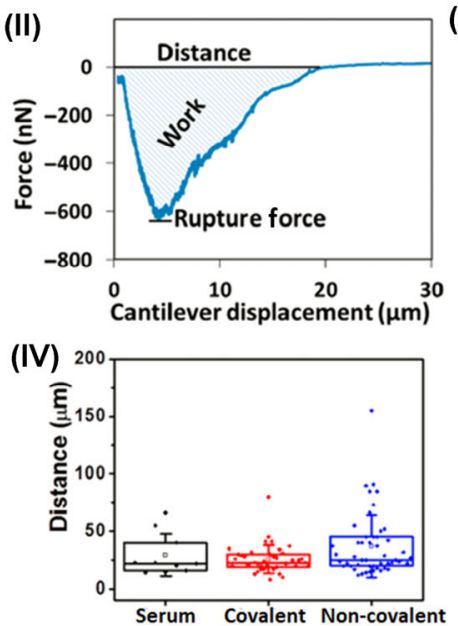

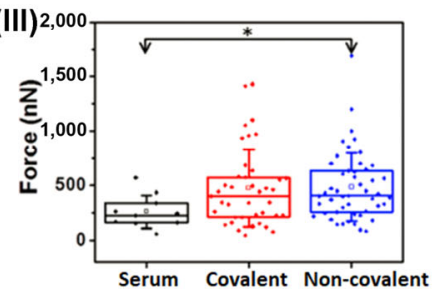

(V)

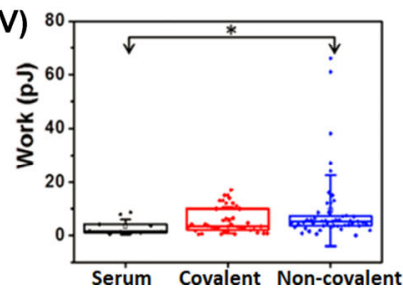

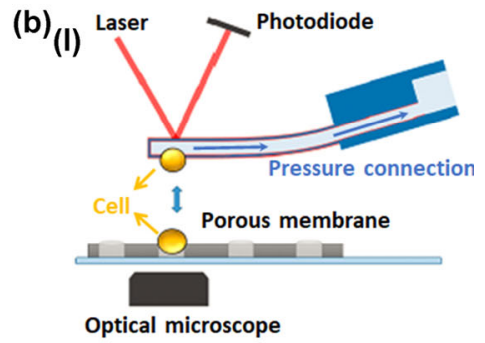

(II)

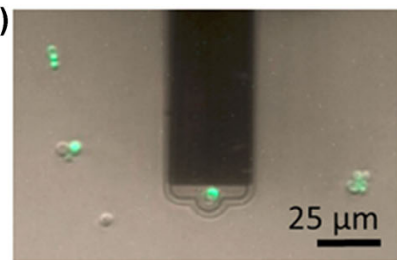

(III)

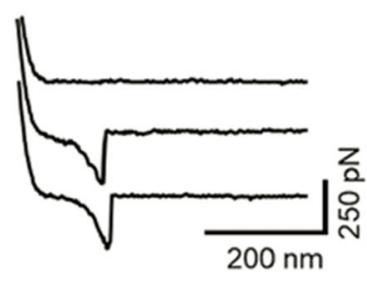

Figure 7 Single-cell adhesion interactions probed by FluidFM. (a) Cell-substrate adhesion interactions. (I) Schematic depicting the FluidFM system for cell-adhesion force spectroscopy on different types of substrates. (II) Representative force curve beginning right after the contact between the cell and the cantilever. The maximum adhesion force is the point where the force to bend the cantilever equals the cell-adhesion force. The total detachment distance required to pull a cell off the surface is measured from the initial point where the cantilever bending begins to when it reaches its original shape again. The area of the shaded region in the force curve represents the work done by the cantilever to detach a cell from the surface and corresponds to the binding energy of a cell with the surface. (III)-(V) Box plots of the (III) maximum adhesion force, (IV) detachment distance, and (V) total work done in the cell pick-up experiments from gold surfaces coated with serum proteins, covalent bound RGD, and non-covalently bound RGD. (Reprinted with permission from Ref. [82], (c) American Chemical Society 2017). (b) Adhesion interactions between yeast cells. (I) Schematic showing probing the adhesion interactions between two yeast cells by FluidFM. (II) Labeling of the attached cell (in green) demonstrates that cell integrity is preserved, thus that the method is nondestructive. (III) Typical force curves (only retraction profiles are shown) obtained during SCFS experiments. (Reprinted with permission from Ref. [95], (c) American Chemical Society 2019).

and macromolecular forces), but so far the precise mechanisms by which these interactions determine cell adhesion processes are not yet fully understood [91]. With the use of FluidFM, single microbe can be easily immobilized to the AFM cantilever by applying underpressure, and then the adhesive interactions between microbes and substrates (or microbes) can be measured. In 2015, with the use of pyramidal microchanneled cantilever, Potthoff et al. [92] firstly showed that FluidFM is able to suck single bacterium and then measure the adhesion forces between bacteria and substrates. In 2017, Sprecher et al. [93] showed that FluidFM-based SCFS could benefit the investigation of the exopolysaccharide adhesins in bacterial surface colonization and biofilm formation. Candida albicans is the principal cause of opportunistic mycoses worldwide and the agglutinin-like sequence (Als) protein on the surface of $C$. albicans is closely related to the adhesion, aggregation and biofilm formation of C. albicans. [94]. In 2019, the studies by Dehullu et al. [95] with the use of FluidFM-based SCFS discovered a previously undescribed function (mediating amyloid-like homophilic adhesion) for C. albicans Als proteins. A yeast cell was immobilized on the aperture of the hollow cantilever by applying a negative pressure, and another yeast cell was mechanically trapped in porous membrane $((\mathrm{I})$ in Fig. 7(b)). Staining the cell with live/dead indicator dye confirmed the integrity of the cell ((II) in Fig. 7(b)). The interactions between yeast cells at different conditions (e.g., altering the amyloid sequence or lowering the protein density on the cell surface) were then measured by performing SCFS to record force curves ((III) in Fig. 7(b)). The results significantly revealed a novel aggregation mechanism of C. albicans, in which force-dependent amyloid core sequences in Als proteins play a dual role, that is, in formation of adhesin nanoclusters and in homophilic bonding between amyloid sequences on opposing cells. Recently, the optimization of measurement parameters (e.g., setpoint, z-speed, z-length, pause time, and relative underpressure) when applying FluidFM-based SCFS on bacterial cells has been investigated [96]. As an alternative way, the bead whose surface is coated with chemical molecules can be attached to the aperture of the microchanneled cantilever, which is then used to probe the interactions between individual microbial cells (immobilized on the substrate) and bead surface [97]. In future, FluidFM may contribute to the identification of small peptide inhibitors [98] and titanium surface [99] for antiadhesion therapy.

\subsection{FluidFM limitations for cell adhesion}

The serial cell-adhesion protocol is without doubt the most straightforward one of those established with the FluidFM because of its broad validity. Nevertheless, it is not immune to drawbacks. If applied to mammalian cells, it is often observed that cell debris remain attached at the aperture edge despite the antifouling coating, which then requires a cleaning step by immersing in an appropriate solution that can take place keeping the FluidFM probe mounted on the AFM head. It works although there is a risk that the cleaning solution may affect the antifouling layer and thus jeopardizes its efficiency. On the other hand, if applied to bacteria, one has to be aware of the compromise between size of the aperture and adhesion 
strength. Bacteria need a small aperture to be efficiently attached at it, yet a small aperture means a small suction force (a few tens on nanoNewtons for an aperture diameter of around $300 \mathrm{~nm}$ and an applied underpressure of maximal 1 atmosphere [25]) sometimes not able to overcome the adhesion strength of the bacteria under investigation.

\section{FluidFM for single-cell electrophysiology}

\subsection{Force-controlled patch clamp}

FluidFM could inspire original developments for patch clamp. Ion channels are a diverse family of membrane-spanning proteins that lower the free energy required for ions to traverse the plasma membrane [100], and ion channels play an important role in regulating the life activities of cells [101]. Malfunctioning of ion channels has been implicated in human diseases (called channelopathies [102]) such as lysosomal storage disorders, neurodegenerative diseases and metabolic pathologies, as well as the progression of certain infectious diseases [103]. The patch clamp technique is an electrophysiological method that allows the recording of macroscopic whole-cell or microscopic single-channel currents in single cells using glass micropipettes filled with an electrolytic solution [104] and patch clamp has been the gold standard of the studies of ion channel for decades [105]. Combining FluidFM with patch clamp techniques allows precise monitoring of the mechanical forces exerted on cells while simultaneously sensing the ionic currents of the cell [106]. For doing this, an electrode was inserted into the reservoir of the FluidFM probe, and another electrode was placed in the bath solution $(150 \mathrm{mM}$ $\mathrm{KCl}$ ) (Fig. 8(a)). A cylindrical aperture with a diameter of about $350 \mathrm{~nm}$ was milled by FIB at the pyramid apex (Fig. 8(b)). After selecting a cell on the dish, the FluidFM probe was positioned on top of it under optical microscopy guidance (Fig. 8(c)) and the probe was approached on the membrane of the targeted cell via the AFM force feedback. By applying a slight underpressure a seal was formed in the cylindrical aperture of around $150 \mathrm{M} \Omega$ allowing simultaneously monitoring ionic current and the force of the nanopipette (Fig. 8(d)). The contact between the tip and the cell is stable due to the automated force feedback of AFM, whereas the contact is prone to get lost in conventional patch clamping due to vibrations or cell volume changes. The currents in response to conventional voltage pulse were recorded and the superimposed whole-cell $\mathrm{Na}^{+}$currents of the $\mathrm{Na}_{\mathrm{v}} 1.5$ channel confirmed the specific electrophysiological characteristics of the $\mathrm{Na}_{\mathrm{v}} 1.5$ ion channel (Fig. 8(e)). Based on the method, the whole-cell ionic currents and the contraction forces of mouse adult cardiomyocytes were simultaneously recorded (Figs. $8(\mathrm{f})$ and $8(\mathrm{~g})$ ), clearly showing the rhythmic movements of the cardiomyocyte as well as the changes of whole-cell ionic currents. Due to the unique advantages of the FluidFM-based patch clamp, it is particularly suited to investigate the behaviors of mechanosensitive ion channels $[107,108]$ which are activated by mechanical forces for revealing the principles of ion-channel-mediated mechanosensory transduction [109].

\subsection{Force-controlled scanning ion conductance microscopy (SICM)}

FluidFM can be also operated in simultaneous SICM mode. So far, it is still challenging for regular AFM to resolve the fine structures of living mammalian cells. First, the very soft and dynamic nature of the cell surfaces makes imaging living mammalian cells difficult [76]. Besides, the mechanical contact between AFM tip and cell surface usually deforms cellular
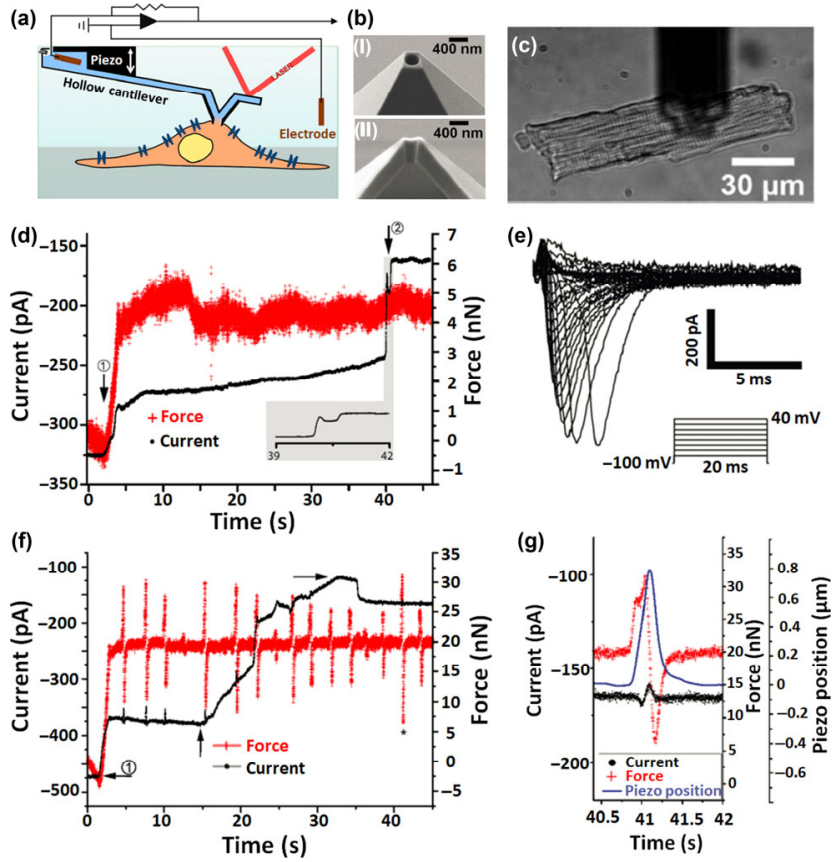

Figure 8 Single-cell patch clamp while simultaneously monitoring the mechanical forces by FluidFM. (a) Schematic of the FluidFM setup in whole-cell configuration. (b) SEM images of the pyramidal tip for patch clamping. (I) Zoom-in of the tip showing the aperture at the apex of the tip. (II) FIB section of the probe. (c) Positioning the FluidFM probe to the targeted cell under the guidance of optical microscopy. (d) Simultaneously recording the whole-cell $\mathrm{Na}_{\mathrm{v}} 1.5$ currents and the mechanical forces exerted on the cell from the probe. The FluidFM probe is lowered to the point of the contact (denoted by the arrow 1) and then the force control maintains the force signal. Little negative pressure is exerted on the fluidic channel of the probe for $40 \mathrm{~s}$ (denoted by the arrow 2) and a seal is obtained for recording whole-cell ionic currents. (e) Sodium currents recording elicited from a series of voltage pulses of $5 \mathrm{mV}$ increments. (f) Simultaneous whole-cell current recordings and contraction force on primary mouse cardiomyocytes. The force increases simultaneously with a drop in ionic current as the FluidFM tip contacts the cell (denoted by arrow 1). Not labeled arrows indicate slight increase of suction force through a $1 \mathrm{~mL}$ syringe. (g) Zoom-in of the contraction marked with an asterisk in (f). In addition, the relative position of the piezo actuator is shown. (Reprinted with permission from Ref. [106], (C) American Chemical Society 2015).

surface molecules, which in turn results in the blurring of the recorded images [110]. To solve this issue, we would need a scanning probe technique in which the probe is not in direct physical contact with the cell surface, which is known as SICM [111]. Studies have shown that on very soft samples such as living cells, SICM can be advantageous to AFM in terms of imaging quality, accuracy in topography measurements, lateral resolution, and long-term imaging stability [112]. SICM uses an electrolyte-filled glass pipette (nanopipette) as a probe and relies on an ion current flowing between an electrode inside the nanopipette and another in an external bath solution [113]. The ionic current passing through the apex opening of the nanopipette is sensitive to the tip-sample separation, allowing non-contact topographic imaging of the specimens. Implementing SICM on the FluidFM allows simultaneously recording mechanical and conductance information of the specimens [114]. An electrode is inserted in the fluidic circuit of the FluidFM, and another electrode is in the bath solution (Fig. 9(a)). The ionic current through the microchanneled cantilever is monitored with a patch clamp amplifier. Under this configuration, controlling the FluidFM probe (Fig. 9(b)) to scan the specimen allows recording simultaneously both the deflection of the cantilever and the ionic current. The topography images of specimens are obtained at non-contact 
(a)

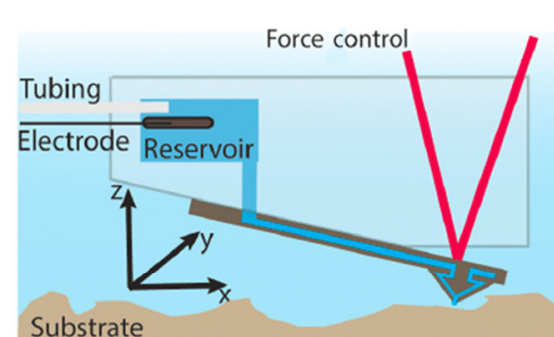

(b)

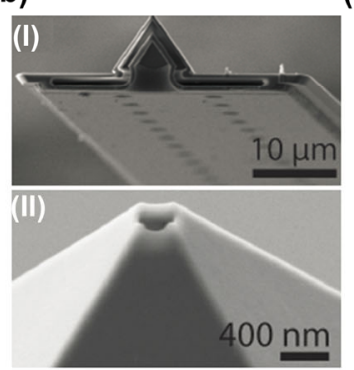

(c) (d) (I)

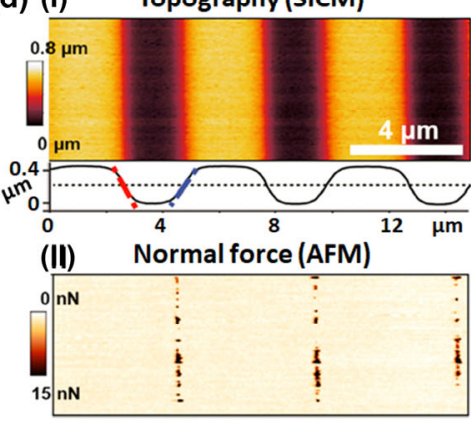

(e)

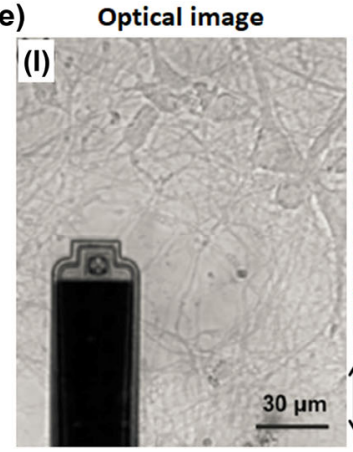

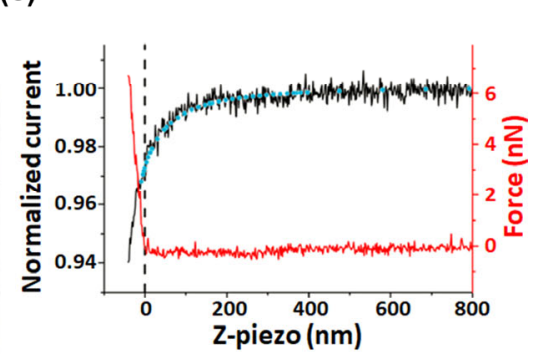

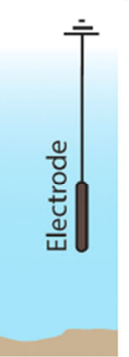

Topography
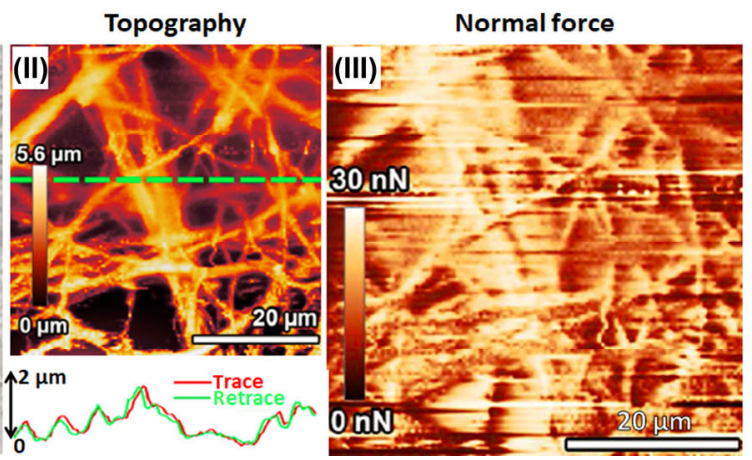

Figure 9 Simultaneous SICM and AFM for cell imaging by FluidFM. (a) Schematic of the FluidFM with Ag/AgCl electrodes for ionic current recording through the embedded microchannel. (b) SEM images of the FIB cross-sectioned microchannel cantilever (I) and the aperture of the probe. (c) Simultaneous force and ionic current spectroscopy. Overlaid on the ionic current curve is the simulated approach curve (blue dots). (d) Simultaneous SICM topography imaging (I) and AFM force imaging (II) on calibration grid which has well-defined geometry. (e) Simultaneous SICM and AFM imaging on living neural cells. (I) Optical image of the FluidFM probe in proximity of the neuron. (II) SICM topography showing the intricate three-dimensional network structures of the living neuron. Trace and retrace profiles taken along the dashed line are shown in the lower part. (III) The corresponding AFM force image of the neuron. (Reprinted with permission from Ref. [114], (c) American Physical Society 2015).

mode by using the ionic current as the feedback, and the force images of specimens are generated simultaneously from the force feedback of the cantilever. Force spectroscopy experiments performed on substrates show the simultaneously obtained ionic curve and force curve (Fig. 9(c)), which delivers mechanical and electrical information. Experiments performed on standard grids (Fig. 9(d)) and living neurons (Fig. 9(e)) simultaneously show the topographic images and force images of specimens. The results [114] significantly show that FluidFM provides novel possibilities to SICM by integrating AFM force feedback, which will be particularly meaningful for investigating the structures and multiple properties (e.g., mechanics and electronics) of single cells.

\subsection{Force-controlled scanning nanopore microscopy}

The aperture at the pyramidal apex of the FluidFM probes can also be interpreted as a nanopore for the stochastic sensing of secreted molecules and the activity of ion channels in arbitrary locations both outside and inside a cell. Observing the activities of single molecules of single living cell in their native states is of critical significance for the investigations of cell signaling and molecular mechanisms [115]. Traditional single-molecule methods require the fluorescent labeling of the targeted molecules [116-118], which may influence the activities of molecules and cells. In fact, strictly speaking, the results obtained by single-molecule fluorescence microscopy only reflect the behaviors of the individual fluorescent spots but not the targeted biomolecules themselves [119]. Asymmetric glass capillary nanopore electrode has been used for singlemolecule detection on single living cells [120], but the measurement precision is limited due to the lack of sensitive feedback. FluidFM can be used as scanning nanopore microscopy to sense the activities of biomolecules using simultaneous force and ion-current feedback [49]. For doing this, the apex of the tip is flattened by ion sputtering to produce a thin film of silicon nitride and then a small hole is formed in the middle of the thin film using focused $\mathrm{He}^{+}$ions (Fig. 10(a)). We can shrink the pore to a desired size by raster scanning of the $\mathrm{He}^{+}$ ions across the pore (Fig. 10(b)). The nanopore provides the nanoscale confinement of biomolecules between the apex and the underlying surface ((I) in Fig. 10(c)) and sets a barrier for the biomolecules before their entrance into the nanopore ((II) in Fig. 10(c)). The current-voltage $(I-V)$ curve of the nanopore near the surface is recorded, which is dependent on the protein translocation through the nanopore, allowing the detection of single biomolecules. With FluidFM-based nanopore, not only the topographical image and the ionic current map of single living cell can be obtained (Fig. 10(e)), but also the proteins secreted by the targeted cell can be real-timely monitored via distinctive blockades and/or enhancement in the current traces (Fig. 10(f)). Besides, FluidFM-based nanopore enabled intracellular recordings at particular parts of the targeted cell (Fig. 10(g)). A large number of current spikes were observed (Fig. 10(h)), indicating the translocation of intracellular elements through the nanopore. Overall, the results [49] showed the great potentials of FluidFM-based scanning nanopore microscopy in addressing biological issues with unprecedented spatial resolution and force/ionic current sensitivity, which will contribute to the studies of single-cell electrophysiology at molecular level.

\subsection{FluidFM limitations for electrophysiology}

If the force feedback of the FluidFM is an advantage with respect to glass capillaries as far as the stability of the contact with the cell membrane, the tough challenge for the FluidFM is to show the capability of consistently obtaining the compulsory gigaseal. The pyramidal tip being not the most indicated for the establishment of the gigaseal due to the corners between the facets, it will be mandatory to explore the microfabrication of hollow tips with a cylindrical symmetry. Concerning the 
(a)
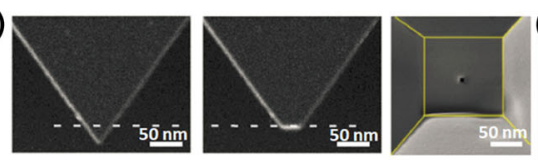

(c)

(b)
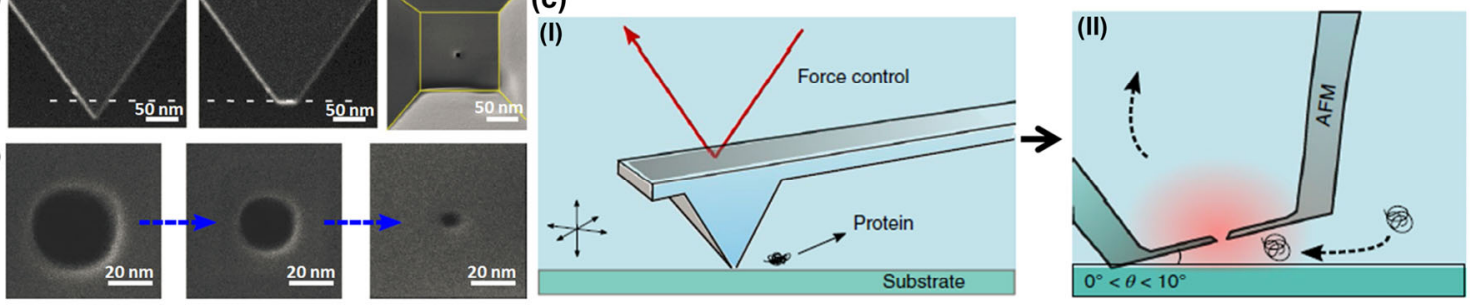

(d)

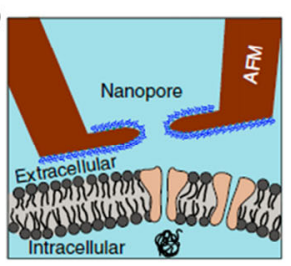

(e)

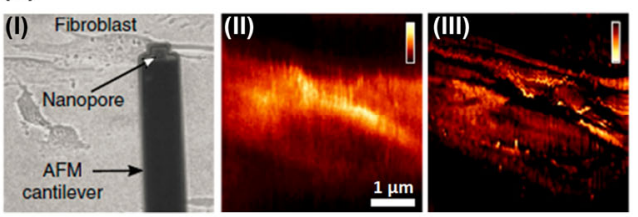

(f)

(g)

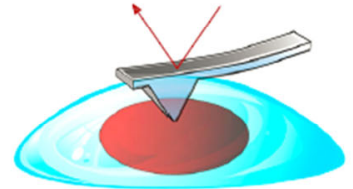

(h)
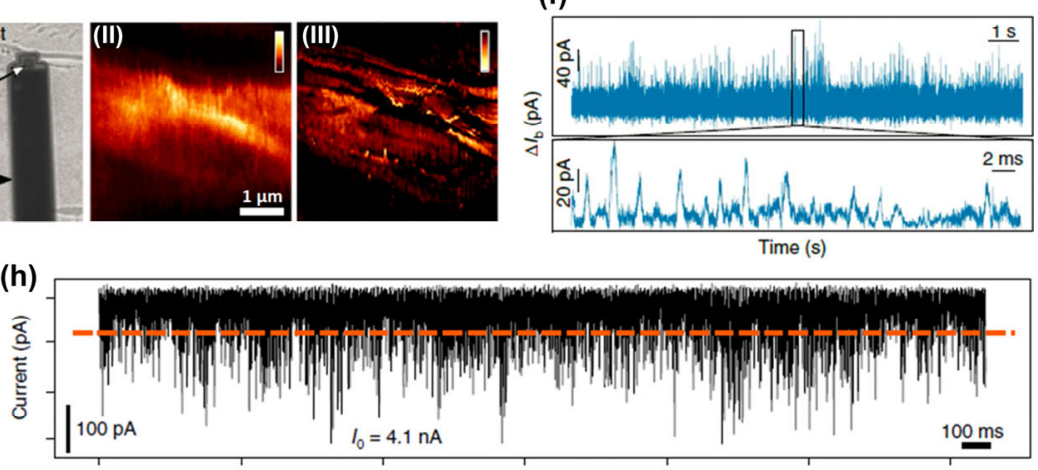

Time (s)

Figure 10 Sensing the biomolecules both outside and inside a cell by FluidFM-based scanning nanopore microscopy. (a) Flattening of the pyramid apex with $\mathrm{Ga}^{+}$irradiation (dashed line), and the subsequent pore drilling with a focused $\mathrm{He}^{+}$ion beam. (b) Ion-beam sculpting process in which the prefabricated pore can be shrunk in diameter by raster scanning of $\mathrm{He}^{+}$ions across the pore. (c) Schematic of the experimental set-up and the concept of protein confinement using mechanical forces. The approach of the tip to the surface (I) creates a nanoconfined area that produces an energy barrier for biomolecular translocation before the entrance to the nanopore (II). (d) Schematic of the FluidFM nanopore on the surface of a cell. (e) For extracellular recording of protein secretion, the FluidFM nanopore is located on top of a fibroblast cell under optical microscopy (I) to simultaneously record topographical image (II) and ionic current maps (III). (f) Current curves recorded on an arbitrary location on top of a fibroblast cell showing the activities of biomolecules secreted by the cell. (g) Schematic of detecting intracellular activities by FluidFM nanopore. (h) Typical current curves recorded inside a cell. The dashed line indicates the detection threshold. (Reprinted with permission from Ref. [49], @ Springer Nature 2019).

nanopore mode, the promising aspect is surely related to the possibility to carry out the measurements close to or even within a cell; nonetheless the huge challenge is to reliably determine the identity of the biomolecules passing through the nanoaperture. Moreover, it has to be established whether translocating biomolecules are released by the approached cell or are from the cell culture medium [121].

\section{Conclusion and future perspectives}

In this review, the achievements in single-cell manipulations and detections using FluidFM in the past decade since its invention have been summarized, considerably demonstrating that FluidFM has become a powerful multifunctional toolbox for single-cell biophysics, including various single-cell manipulations (e.g., three-dimensional operation [48], cell sorting [56], cell patterning [57], exchangeable colloidal probe for cell indenting [65], drug dispensing \& injection with very small volumes [25], cell nucleus injection [68], individual virus stimulation [67], and cellular extraction [70]), single-cell force spectroscopy (e.g., cell-substrate interaction measurements [82] and cell-cell interaction measurements [95]), and single-cell electrophysiology (e.g., simultaneous mechanical force and ionic current recording for patch clamp [106], force-controlled SICM [114], and single-molecule sensing based on scanning nanopore microscopy [49]), providing novel possibilities for investigating the behaviors of single living cells, which is significantly meaningful for the studies of revealing the underlying molecular mechanisms guiding cellular physiological/pathological processes.

\subsection{Technical challenges and developments}

There is still considerable room for the advancement of
FluidFM. Notably, at present, the three-dimensional manipulations by FluidFM (via controlling the pressure of the fluid channel in the FluidFM probe) are dependent on the guidance of optical microscopy. For the objects which are invisible for optical microscopy, the objects being manipulated by FluidFM need to be labeled with fluorescence for visual recognition; for example, when manipulating single virus, the virus was labeled with fluorescein [67]. Besides, when applying FluidFM to deliver/ inject liquids to single cells [56] or to extract intracellular ingredients [70], fluorescent dyes are mixed in the solution or cells are engineered to express GFP for visually exact determination of the volumes of the delivered liquids or the extractions. The introduction of fluorescein may influence the activities of the cells or the objects being delivered and therefore the conditions are not fully native. Studies have shown the successful label-free optical imaging of 75-nm adenoviruses by submerged microsphere optical nanoscopy without the use of fluorescent labeling [122], which is inaccessible for conventional optical microscopy. Therefore, introducing the advanced microsphere-aided super-resolution optical imaging to the optical system of FluidFM will help improving the label-free manipulations of FluidFM. For FluidFM-based whole-cell patch clamping, the current seal resistances achieved are typically less than $150 \mathrm{M} \Omega$ [106] which is insufficient for reaching gigaseal, significantly influencing the measurement quality of patch clamping. Optimizing the geometry of the FluidFM probes will be mandatory to improve the seal resistance for patch clamp [52].

\subsection{Future directions for FluidFM-based single-cell biophysics}

Further explorations using FluidFM will contribute to answering fundamental issues involved in biomechanics at the single-cell 
level. So far in practice for standard AFM-based studies of cell mechanics in response to external chemical stimuli, drug molecules are added into the dish perturbating all the cells on the dish [32], making impossible to estimate how many drug molecules are indeed acting on the targeted cell. With FluidFM, we can precisely deliver known volume of drug molecules to the selected cell on the dish [56] and then monitor the realtime mechanical changes of the targeted cell (specially, due to the fact that the hollow tip is not suited for AFM indentation assays, single microsphere can be immobilized to the aperture of FluidFM probe for measurement [51] prior cellular mechanical measurements). Particularly, since the delivery is locally for the targeted cell whereas the other cells are probably not influenced by the delivered drug molecules, it is very convenient for control experiments; for example, we can perform measurements on the cells that are not influenced by the drug molecules in the same dish. Since the measurements are performed in identical conditions (e.g., in the same dish, in the same cell culture medium, and in the same environment), it fully meets the requirements of comparative studies [123]. These experiments will be particularly useful for revealing the detailed dynamics of cell mechanics taking place after the treatment of known small volumes of drug molecules on single cells. Besides, with the use of FluidFM's three-dimensional capabilities, cells can be picked up and placed on the different substrates with diverse properties (such as stiffness) for measurements, and therefore this will significantly facilitate examining the exquisite effects of substrate properties on the cellular mechanics (e.g., elasticity, viscoelasticity, and adhesion force [124]) at the single same cell level, which is quite meaningful for the studies of cell mechanics.

Due to the intrinsic geometry of the apex of the hollow probes, FluidFM is not suited for high-resolution morphological imaging of the samples. Combining FluidFM with complementary techniques will benefit the investigation of the activities of single cells with subcellular resolution. Based on the single-cell manipulation of FluidFM, intracellular substances of single living cells can be extracted for further biochemical assays [70], which can provide the chemical properties of the cell. Besides, we can use regular AFM high-resolution morphological imaging and mechanical analysis [28] to characterize these intracellular structures. So far studies of organelles by AFM were performed on organelles (e.g., cell mitochondria [125] and cell nucleus [126]) which are isolated from the lysis of the ensembles of cells and therefore the results only reflect the averaged behavior of organelles from cell populations. Combining FluidFM with regular AFM as well as biochemical assays, we can probe the activities of single living targeted cell, after which the structures and properties (mechanics and chemical properties) of the organelles of the targeted cell can be characterized, thus providing a feasible way for correlating the structures and properties of organelles with the behaviors of the same cell with subcellular resolution. The origin and spread of molecular mutations take place at multiple scales (from the organelle to cell and the human population) [127] and recently researchers have shown the great significance of investigating enzymatic activities in organelles for discovering novel mechanisms guiding critical cellular functions [128]. Consequently, combining FluidFM with complementary techniques to investigate cell behaviors at subcellular resolution will have general impacts on the studies of life sciences.

Taken together, the developments of FluidFM significantly provide novel possibilities for single-cell biophysics, which will have great impacts on the communities of biomedicine. Notably FluidFM for biomedical applications is still in its infancy and there is huge room for further advancements.
In the future, as more biological systems are investigated by FluidFM, particularly combined with other complementary techniques, we have much to look forward to.

\section{Acknowledgements}

This work was supported by the China Scholarship Council (CSC) (No. 202004910157). M. Li is thankful for the support from the National Natural Science Foundation of China (Nos. 61922081 and 61873258), the Key Research Program of Frontier Sciences CAS (No. ZDBS-LY-JSC043), the Youth Innovation Promotion Association CAS (No. 2017243), and the LiaoNing Revitalization Talents Program (No. XLYC1907072).

Funding note: Open Access funding provided by Swiss Federal Institute of Technology Zurich

Open Access This article is licensed under a Creative Commons Attribution 4.0 International License, which permits use, sharing, adaptation, distribution and reproduction in any medium or format, as long as you give appropriate credit to the original author(s) and the source, provide a link to the Creative Commons licence, and indicate if changes were made.

The images or other third party material in this article are included in the article's Creative Commons licence, unless indicated otherwise in a credit line to the material. If material is not included in the article's Creative Commons licence and your intended use is not permitted by statutory regulation or exceeds the permitted use, you will need to obtain permission directly from the copyright holder.

To view a copy of this licence, visit http://creativecommons.org/licenses/by/4.0/.

\section{References}

[1] Altschuler, S. J.; Wu, L. F. Cellular heterogeneity: Do differences make a difference? Cell 2010, 141, 559-563.

[2] Pennisi, E. Single-cell sequencing tackles basic and biomedical questions. Science 2012, 336, 976-977.

[3] Pelkmans, L. Using cell-to-cell variability-A new era in molecular biology. Science 2012, 336, 425-426.

[4] Ackermann, M. A functional perspective on phenotypic heterogeneity in microorganisms. Nat. Rev. Microbiol. 2015, 13, 497-508.

[5] Raj, A.; van Oudenaarden, A. Nature, nurture, or chance: Stochastic gene expression and its consequences. Cell 2008, 135, 216-226.

[6] Marusyk, A.; Almendro, V.; Polyak, K. Intra-tumour heterogeneity: A looking glass for cancer? Nat. Rev. Cancer 2012, 12, 323-334.

[7] Burrell, R. A.; McGranahan, N.; Bartek, J.; Swanton, C. The causes and consequences of genetic heterogeneity in cancer evolution. Nature 2013, 501, 338-345.

[8] Bedard, P. L.; Hansen, A. R.; Ratain, M. J.; Siu, L. L. Tumour heterogeneity in the clinic. Nature 2013, 501, 355-364.

[9] Stuart, T.; Satija, R. Integrative single-cell analysis. Nat. Rev. Genet. 2019, 20, 257-272.

[10] Wu, A. R.; Neff, N. F.; Kalisky, T.; Dalerba, P.; Treutlein, B.; Rothenberg, M. E.; Mburu, F. M.; Mantalas, G. L.; Sim, S.; Clarke, M. F. et al. Quantitative assessment of single-cell RNA-sequencing methods. Nat. Methods 2014, 11, 41-46.

[11] Karemaker, I. D.; Vermeulen, M. Single-cell DNA methylation profiling: Technologies and biological applications. Trends Biotechnol. 2018, 36, 952-965.

[12] Kang, C. C.; Yamauchi, K. A.; Vlassakis, J.; Sinkala, E.; Duncombe, T. A.; Herr, A. E. Single cell-resolution western blotting. Nat. Protoc. 2016, 11, 1508-1530.

[13] Taheri-Araghi, S.; Brown, S. D.; Sauls, J. T.; McIntosh, D. B.; Jun, S. Sigle-cell physiology. Ann. Rev. Biophys. 2015, 44, 123-142.

[14] Patel, A. P.; Tirosh, I.; Trombetta, J. J.; Shalek, A. K.; Gillespie, S. M.; Wakimoto, H.; Cahill, D. P.; Nahed, B. V.; Curry, W. T.; Martuza, R. L. et al. Single-cell RNA-seq highlights intratumoral heterogeneity in primary glioblastoma. Science 2014, 344, 1396-1401. 
[15] Deng, Q. L.; Ramsköld, D.; Reinius, B.; Sandberg, R. Single-cell RNA-seq reveals dynamic, random monoallelic gene expression in mammalian cells. Science 2014, 343, 193-196.

[16] Luquette, L. J.; Bohrson, C. L.; Sherman, M. A.; Park, P. J. Identification of somatic mutations in single cell DNA-seq using a spatial model of allelic imbalance. Nat. Commun. 2019, 10, 3908.

[17] Dumont, S.; Prakash, M. Emergent mechanics of biological structures. Mol. Biol. Cell 2014, 25, 3461-3465.

[18] Janmey, P. A.; McCulloch, C. A. Cell mechanics: Integrating cell responses to mechanical stimuli. Annu. Rev. Biomed. Eng. 2007, 9, $1-34$.

[19] Kim, D. H.; Wong, P. K.; Park, J.; Levchenko, A.; Sun, Y. Microengineered platforms for cell mechanobiology. Annu. Rev. Biomed. Eng. 2009, 11, 203-233.

[20] Suresh, S. Biomechanics and biophysics of cancer cells. Acta Biomater. 2007, 3, 413-438.

[21] Wirtz, D.; Konstantopoulos, K.; Searson, P. C. The physics of cancer: The role of physical interactions and mechanical forces in metastasis. Nat. Rev. Cancer 2011, 11, 512-522.

[22] Mitchell, M. J.; Jain, R. K.; Langer, R. Engineering and physical sciences in oncology: Challenges and opportunities. Nat. Rev. Cancer 2017, 17, 659-675.

[23] Yamauchi, K. A.; Herr, A. E. Subcellular western blotting of single cells. Microsyst. Nanoeng. 2017, 3, 16079.

[24] Yu, W. B.; Sharma, S.; Gimzewski, J. K.; Rao, J. Y. Nanocytology as a potential biomarker for cancer. Biomark. Med. 2017, 11, 213-216.

[25] Meister, A.; Gabi, M.; Behr, P.; Studer, P.; Vörös, J.; Niedermann, P.; Bitterli, J.; Polesel-Maris, J.; Liley, M.; Heinzelmann, H. et al. FluidFM: Combining atomic force microscopy and nanofluidics in a universal liquid delivery system for single cell applications and beyond. Nano Lett. 2009, 9, 2501-2507.

[26] Binnig, G.; Quate, C. F.; Gerber, C. Atomic force microscope. Phys. Rev. Lett. 1986, 56, 930-933.

[27] Li, M.; Xi, N.; Wang, Y. C.; Liu, L. Q. Advances in atomic force microscopy for single-cell analysis. Nano Res. 2019, 12, 703-718.

[28] Dufrêne, Y. F.; Ando, T.; Garcia, R.; Alsteens, D.; Martinez-Martin, D.; Engel, A.; Gerber, C.; Muller, D. J. Imaging modes of atomic force microscopy for application in molecular and cell biology. Nat. Nanotechnol. 2017, 12, 295-307.

[29] Krieg, M.; Fläschner, G.; Alsteens, D.; Gaub, B. M.; Roos, W. H.; Wuite, G. J. L.; Gaub, H. E.; Gerber, C.; Dufrêne, Y. F.; Muller, D. J. Atomic force microscopy-based mechanobiology. Nat. Rev. Phys. 2019, 1, 41-57.

[30] Di Carlo, D. A mechanical biomarker of cell state in medicine. J. Lab. Autom. 2012, 17, 32-42.

[31] Moeendarbary, E.; Harris, A. R. Cell mechanics: Principles, practices, and prospects. WIREs Syst. Biol. Med. 2014, 6, 371-388.

[32] Li, M.; Dang, D.; Liu, L. Q.; Xi, N.; Wang Y. C. Atomic force microscopy in characterizing cell mechanics for biomedical applications: A review. IEEE Trans. NanoBiosci. 2017, 16, 523-540.

[33] Harris, A. R.; Peter, L.; Bellis, J.; Baum, B.; Kabla, A. J.; Charras, G. T. Characterizing the mechanics of cultured cell monolayers. Proc. Natl. Acad. Sci. USA 2012, 109, 16449-16454.

[34] Wu, P. H.; Aroush, D. R. B.; Asnacios, A.; Chen, W. C.; Dokukin, M. E.; Doss, B. L.; Durand-Smet, P.; Ekpenyong, A.; Guck, J.; Guz, N. V. et al. A comparison of methods to assess cell mechanical properties. Nat. Methods 2018, 15, 491-498.

[35] Cross, S. E.; Jin, Y. S.; Rao, J. Y.; Gimzewski, J. K. Nanomechanical analysis of cells from cancer patients. Nat. Nanotechnol. 2007, 2, 780-783.

[36] Plodinec, M.; Loparic, M.; Monnier, C. A.; Obermann, E. C.; Zanetti-Dallenbach, R.; Oertle, P.; Hyotyla, J. T.; Aebi, U.; BentiresAlj, M.; Lim, R. Y. H.; Schoenenberger, C. A. The nanomechanical signature of breast cancer. Nat. Nanotechnol. 2012, 7, 757-765.

[37] Gavara, N.; Chadwick, R. S. Determination of the elastic moduli of thin samples and adherent cells using conical atomic force microscope tips. Nat. Nanotechnol. 2012, 7, 733-736.

[38] Garcia, R. Nanomechanical mapping of soft materials with the atomic force microscope: Methods, theory and applications. Chem. Soc. Rev. 2020, 49, 5850-5884.

[39] Song, B.; Yang, R. G.; Xi, N.; Patterson, K. C.; Qu, C. G.; Lai, K. W. C.
Cellular-level surgery using nano robots. J. Lab. Autom. 2012, 17, 425-434.

[40] Han, S. W.; Nakamura, C.; Obataya, I.; Nakamura, N.; Miyake, J. A molecular delivery system by using AFM and nanoneedle. Biosens. Bioelectron. 2005, 20, 2120-2125.

[41] Chen, X.; Kis, A.; Zettl, A.; Bertozzi, C. R. A cell nanoinjector based on carbon nanotubes. Proc. Natl. Acad. Sci. USA 2007, 104, 8218-8222.

[42] Hochmuth, R. M. Micropipette aspiration of living cells. J. Biomech. 2000, 33, 15-22.

[43] Zambelli, T.; Aebersold, M. J.; Behr, P.; Han, H.; Hirt, L.; Martinez, V.; Guillaume-Gentil, O.; Vörös, J. FluidFM: Development of the instrument as well as its applications for 2D and 3D lithography. In Open-Space Microfluidics: Concepts, Implementations, Applications. Delamarche, E.; Kaigala, G. V., Eds.; Wiley-VCH Verlag GmbH \& Co. KGaA: Weinheim, 2018; pp 295-323.

[44] Saha, P.; Duanis-Assaf, T.; Reches, M. Fundamentals and applications of FluidFM technology in single-cell studies. Adv. Mater. Interfaces 2020, 7, 2001115.

[45] Li, M.; Xi, N.; Wang, Y. C.; Liu, L. Q. Atomic force microscopy for revealing micro/nanoscale mechanics in tumor metastasis: From single cells to microenvironmental cues. Acta Pharmacol. Sin. 2021, 42, 323-339.

[46] Deladi, S.; Tas, N. R.; Berenschot, J. W.; Krijnen, G. J. M.; de Boer, M. J.; de Boer, J. H.; Peter, M.; Elwenspoek, M. C. Micromachined fountain pen for atomic force microscope-based nanopatterning. Appl. Phys. Lett. 2004, 85, 5361-5363.

[47] Berenschot, E. J. W.; Burouni, N.; Schurink, B.; van Honschoten, J. W.; Sanders, R. G. P.; Truckenmuller, R.; Jansen, H. V.; Elwenspoek, M. C.; van Apeldoorn, A. A.; Tas, N. R. 3D nanofabrication of fluidic components by corner lithography. Small 2012, 8, 3823-3831.

[48] Guillaume-Gentil, O.; Potthoff, E.; Ossola, D.; Franz, C. M.; Zambelli, T.; Vorholt, J. A. Force-controlled manipulation of single cells: From AFM to FluidFM. Trends Biotechnol. 2014, 32, 381-388.

[49] Aramesh, M.; Forró, C.; Dorwling-Carter, L.; Lüchtefeld, I.; Schlotter, T.; Ihle, S. J.; Shorubalko, I.; Hosseini, V.; Momotenko, D.; Zambelli, T. et al. Localized detection of ions and biomolecules with a force-controlled scanning nanopore microscope. Nat. Nanotechnol. 2019, 14, 791-798.

[50] Martinez, V.; Behr, P.; Drechsler, U.; Polesel-Maris, J.; Potthoff, E.; Vörös, J.; Zambelli, T. SU-8 hollow cantilevers for AFM cell adhesion studies. J. Micromech. Microeng. 2016, 26, 055006.

[51] Helfricht, N.; Mark, A.; Dorwling-Carter, L.; Zambelli, T.; Papastavrou, G. Extending the limits of direct force measurements: Colloidal probes from sub-micron particles. Nanoscale 2017, 9 , 9491-9501.

[52] Guillaume-Gentil, O.; Mittelviefhaus, M.; Dorwling-Carter, L.; Zambelli, T.; Vorholt, J. A. FluidFM applications in single-cell biology. In Open-Space Microfluidics: Concepts, Implementations, Applications. Delamarche, E.; Kaigala, G. V., Eds.; Wiley-VCH Verlag GmbH \& Co. KGaA: Weinheim, 2018; pp 325-354.

[53] Konradi, R.; Acikgoz, C.; Textor, M. Polyoxazolines for nonfouling surface coatings-a direct comparison to the gold standard PEG. Macromol. Rapid Commun. 2012, 33, 1663-1676.

[54] Weydert, S.; Zürcher, S.; Tanner, S.; Zhang, N.; Ritter, R.; Peter, T.; Aebersold, M. J.; Thompson-Steckel, G.; Forró, C. et al. Easy to apply polyoxazoline-based coating for precise and long-term control of neural patterns. Langmuir 2017, 33, 8594-8605.

[55] Schlotter, T.; Weaver, S.; Forró, C.; Momotenko, D.; Vörös, J.; Zambelli, T.; Aramesh, M. Force-controlled formation of dynamic nanopores for single-biomolecule sensing and single-cell secretomics. ACS Nano 2020, 14, 12993-13003.

[56] Guillaume-Gentil, O.; Zambelli, T.; Vorholt, J. A. Isolation of single mammalian cells from adherent cultures by fluidic force microscopy. Lab Chip 2014, 14, 402-414.

[57] Martinez, V.; Forró, C.; Weydert, S.; Aebersold, M. J.; Dermutz, H.; Guillaume-Gentil, O.; Zambelli, T.; Vörös, J.; Demkó, L. Controlled single-cell deposition and patterning by highly flexible hollow cantilevers. Lab Chip 2016, 16, 1663-1674.

[58] Collins, D. J.; Morahan, B.; Garcia-Bustos, J.; Doerig, C.; Plebanski, M.; Neild, A. Two-dimensional single-cell patterning with one cell per well driven by surface acoustic waves. Nat. Commun. 2015, 6, 8686. 
[59] Xue, X. F.; Sun, Y. B.; Resto-Irizarry, A. M.; Yuan, Y.; Yong, K. M. A.; Zheng, Y.; Weng, S. N.; Shao, Y.; Chai, Y. M.; Studer, L. et al. Mechanics-guided embryonic patterning of neuroectoderm tissue from human pluripotent stem cells. Nat. Mater. 2018, 17, 633-641.

[60] Li, Q. S., Lee, G. Y. H., Ong, C. N., Lim, C. T. AFM indentation study of breast cancer cells. Biochem. Biophys. Res. Commun. 2008, 374, 609-613.

[61] Li, M.; Liu, L. Q.; Xi, N.; Wang, Y. C.; Dong, Z. L.; Xiao, X. B.; Zhang, W. J. Atomic force microscopy imaging and mechanical properties measurement of red blood cells and aggressive cancer cells. Sci. China Life Sci. 2012, 55, 968-973.

[62] Dimitriadis, E. K.; Horkay, F.; Maresca, J.; Kachar, B.; Chadwick, R. S. Determination of elastic moduli of thin layers of soft material using the atomic force microscope. Biophys. J. 2002, 82, 27982810.

[63] Hu, H.; Shi, B.; Breslin, C. M.; Gignac, L.; Peng, Y. T. A sub-micron spherical atomic force microscopic tip for surface measurements. Langmuir 2020, 36, 7861-7867.

[64] Dörig, P.; Ossola, D.; Truong, A. M.; Graf, M.; Stauffer, F.; Vörös, J.; Zambelli, T. Exchangeable colloidal AFM probes for the quantification of irreversible and long-term interactions. Biophys. J. 2013, 105, 463-472.

[65] Lüchtefeld, I.; Bartolozzi, A.; Morales, J. M.; Dobre, O.; Basso, M.; Zambelli, T.; Vassalli, M. Elasticity spectra as a tool to investigate actin cortex mechanics. J. Nanobiotechnol. 2020, 18, 147.

[66] Hinterdorfer, P.; Dufrêne, Y. F. Detection and localization of single molecular recognition events using atomic force microscopy. Nat. Methods 2006, 3, 347-355.

[67] Stiefel, P.; Schmidt, F. I.; Dörig, P.; Behr, P.; Zambelli, T.; Vorholt, J. A.; Mercer, J. Cooperative vaccinia infection demonstrated at the single-cell level using FluidFM. Nano Lett. 2012, 12, 4219-4227.

[68] Guillaume-Gentil, O.; Potthoff, E.; Ossola, D.; Dörig, P.; Zambelli, T.; Vorholt, J. A. Force-controlled fluidic injection into single cell nuclei. Small 2013, 9, 1904-1907.

[69] Liu, H. J.; Wen, J.; Xiao, Y.; Liu, J.; Hopyan, S.; Radisic, M.; Simmons, C. A.; Sun, Y. In situ mechanical characterization of the cell nucleus by atomic force microscopy. ACS Nano 2014, 8, 3821-3828.

[70] Guillaume-Gentil, O.; Grindberg, R. V.; Kooger, R.; Dorwling-Carter, L.; Martinez, V.; Ossola, D.; Pilhofer, M.; Zambelli, T.; Vorholt, J. A. Tunable single-cell extraction for molecular analyses. Cell 2016, $166,506-516$.

[71] Guillaume-Gentil, O.; Rey, T.; Kiefer, P.; Ibáñez, A. J.; Steinhoff, R.; Brönnimann, R.; Dorwling-Carter, L.; Zambelli, T.; Zenobi, R.; Vorholt, J. A. Single-cell mass spectrometry of metabolites extracted from live cells by fluidic force microscopy. Anal. Chem. 2017, 89, 50175023.

[72] Weaver, V. M. Cell and tissue mechanics: The new cell biology frontier. Mol. Biol. Cell 2017, 28, 1815-1818.

[73] Gardel, M. L. Moving beyond molecular mechanisms. J. Cell Biol. 2015, 208, 143-145.

[74] Helenius, J.; Heisenberg, C. P.; Gaub, H. E.; Muller, D. J. Singlecell force spectroscopy. J. Cell Sci. 2008, 121, 1785-1791.

[75] Friedrichs, J.; Helenius, J.; Muller, D. J. Quantifying cellular adhesion to extracellular matrix components by single-cell force spectroscopy. Nat. Protoc. 2010, 5, 1353-1361.

[76] Müller, D. J.; Dufrêne, Y. F. Atomic force microscopy: A nanoscopic window on the cell surface. Trends Cell Biol. 2011, 21, 461-469.

[77] Dufrêne, Y. F. Atomic force microscopy and chemical force microscopy of microbial cells. Nat. Protoc. 2008, 3, 1132-1138.

[78] Dehullu, J.; Vorholt, J. A.; Lipke, P. N.; Dufrene, Y. F. Fluidic force microscopy captures amyloid bonds between microbial cells. Trends Microbiol. 2019, 27, 728-730.

[79] Potthoff, E.; Guillaume-Gentil, O.; Ossola, D.; Polesel-Maris, J.; LeibundGut-Landmann, S.; Zambelli, T.; Vorholt, J. A. Rapid and serial quantification of adhesion forces of yeast and mammalian cells. PLoS One 2012, 7, e52712.

[80] Potthoff, E.; Franco, D.; D’Alessandro, V.; Starck, C.; Falk, V.; Zambelli, T.; Vorholt, J. A.; Poulikakos, D.; Ferrari, A. Toward a rational design of surface textures promoting endothelialization. Nano Lett. 2014, 14, 1069-1079.

[81] McGrath, J. S.; Quist, J.; Seddon, J. R. T.; Lai, S. C. S.; Lemay, S. G.; Bridle, H. L. Deformability assessment of waterborne protozoa using a microfluidic-enabled force microscopy probe. PLoS One 2016, 11, e0150438.

[82] Sankaran, S.; Jaatinen, L.; Brinkmann, J.; Zambelli, T.; Vörös, J.; Jonkheijm, P. Cell adhesion on dynamic supramolecular surfaces probed by fluid force microscopy-based single-cell force spectroscopy. ACS Nano 2017, 11, 3867-3874.

[83] Jaatinen, L.; Young, E.; Hyttinen, J.; Vörös, J.; Zambelli, T.; Demkó, L. Quantifying the effect of electric current on cell adhesion studied by single-cell force spectroscopy. Biointerphases 2016, 11, 011004.

[84] Sancho, A.; Vandersmissen, I.; Craps, S.; Luttun, A.; Groll, J. A new strategy to measure intercellular adhesion forces in mature cell-cell contacts. Sci. Rep. 2017, 7, 46152.

[85] Cohen, N.; Sarkar, S.; Hondroulis, E.; Sabhachandani, P.; Konry, T. Quantification of intercellular adhesion forces measured by fluid force microscopy. Talanta 2017, 174, 409-413.

[86] Ryma, M.; Blöhbaum, J.; Singh, R.; Sancho, A.; Matuszak, J.; Cicha, I.; Groll, J. Easy-to-prepare coating of standard cell culture dishes for cell-sheet engineering using aqueous solutions of poly (2-n-propyloxazoline). ACS Biomater. Sci. Eng. 2019, 5, 1509-1517.

[87] Wysotzki, P.; Sancho, A.; Gimsa, J.; Groll, J. A comparative analysis of detachment forces and energies in initial and mature cell-material interaction. Colloids Surf B Biointerfaces 2020, 190, 110894.

[88] Sztilkovics, M.; Gerecsei, T.; Peter, B.; Saftics, A.; Kurunczi, S.; Szekacs, I.; Szabo, B.; Horvath, R. Single-cell adhesion force kinetics of cell populations from combined label-free optical biosensor and robotic fluidic force microscopy. Sci. Rep. 2020, 10, 61.

[89] Veerachamy, S.; Yarlagadda, T.; Manivasagam, G.; Yarlagadda, P. K. Bacterial adherence and biofilm formation on medical implants: A review. Proc. Inst. Mech. Eng. H J. Eng. Med. 2014, 228, 1083-1099.

[90] Arciola, C. R.; Campoccia, D.; Montanaro, L. Implant infections: Adhesion, biofilm formation and immune evasion. Nat. Rev. Microbiol. 2018, 16, 397-409.

[91] Dufrêne, Y. F. Sticky microbes: Forces in microbial cell adhesion. Trends Microbiol. 2015, 23, 376-382.

[92] Potthoff, E.; Ossola, D.; Zambelli, T.; Vorholt, J. A. Bacterial adhesion force quantification by fluidic force microscopy. Nanoscale 2015, 7 , 4070-4079.

[93] Sprecher, K. S.; Hug, I.; Nesper, J.; Potthoff, E.; Mahi, M. A.; Sangermani, M.; Kaever, V.; Schwede, T.; Vorholt, J.; Jenal, U. Cohesive properties of the Caulobacter crescentus holdfast adhesin are regulated by a novel c-di-GMP effector protein. mBio 2017, 8 , e00294-17.

[94] Hoyer, L. L.; Cota, E. Candida albicans agglutinin-like sequence (Als) family vignettes: A review of Als protein structure and function. Front. Microbiol. 2016, 7, 280.

[95] Dehullu, J.; Valotteau, C.; Herman-Bausier, P.; Garcia-Sherman, M.; Mittelviefhaus, M.; Vorholt, J. A.; Lipke, P. N.; Dufrene, Y. F. Fluidic force microscopy demonstrates that homophilic adhesion by Candida albicans Als proteins is mediated by amyloid bonds between cells. Nano Lett. 2019, 19, 3846-3853.

[96] Hofherr, L.; Müller-Renno, C.; Ziegler, C. FluidFM as a tool to study adhesion forces of bacteria-optimization of parameters and comparison to conventional bacterial probe scanning force spectroscopy. PLoS One 2020, 15, e0227395.

[97] Mittelviefhaus, M.; Müller, D. B.; Zambelli, T.; Vorholt, J. A. A modular atomic force microscopy approach reveals a large range of hydrophobic adhesion forces among bacterial members of the leaf microbiota. ISME J. 2019, 13, 1878-1882.

[98] Mathelié-Guinlet, M.; Viela, F.; Viljoen, A.; Dehullu, J.; Dufrêne, Y. F. Single-molecule atomic force microscopy studies of microbial pathogens. Curr. Opin. Biomed. Eng. 2019, 12, 1-7.

[99] Doll, K.; Yang, I.; Fadeeva, E.; Kommerein, N.; Szafrański, S. P.; der Wieden, G. B.; Greuling, A.; Winkel, A.; Chichkov, B. N.; Stumpp, N. S.; Stiesch, M. Liquid-infused structured titanium surfaces: Antiadhesive mechanism to repel Streptococcus oralis biofilms. ACS Appl. Mater. Interfaces 2019, 11, 23026-23038.

[100] Dunlop, J.; Bowlby, M.; Peri, R.; Vasilyev, D.; Arias, R. Highthroughput electrophysiology: An emerging paradigm for ion-channel screening and physiology. Nat. Rev. Drug Discov. 2008, 7, 358-368.

[101] Becchetti, A.; Petroni, G.; Arcangeli, A. Ion channel conformations regulate integrin-dependent signaling. Trends Cell Biol. 2019, 29, 298-307. 
[102] Zaydman, M. A.; Silva, J. R.; Cui, J. M. Ion channel associated diseases: Overview of molecular mechanisms. Chem. Rev. 2012, 112, 6319-6333.

[103] Chen, C. C.; Cang, C. L.; Fenske, S.; Butz, E.; Chao, Y. K.; Biel, M.; Ren, D. J.; Wahl-Schott, C.; Grimm, C. Patch-clamp technique to characterize ion channels in enlarged individual endolysosomes. Nat. Protoc. 2017, 12, 1639-1658.

[104] Annecchino, L. A.; Morris, A. R.; Copeland, C. S.; Agabi, O. E.; Chadderton, P.; Schultz, S. R. Robotic automation of in vivo twophoton targeted whole-cell patch-clamp electrophysiology. Neuron 2017, 95, 1048-1055.e3.

[105] Obergrussberger, A.; Goetze, T. A.; Brinkwirth, N.; Becker, N.; Friis, S.; Rapedius, M.; Haarmann, C.; Rinke-Weiß, I.; Stölzle-Feix, S.; Brüggemann, A. et al. An update on the advancing high-throughput screening techniques for patch clamp-based ion channel screens: Implications for drug discovery. Expert Opin. Drug Discov. 2018, 13, 269-277.

[106] Ossola, D.; Amarouch, M. Y.; Behr, P.; Vörös, J.; Abriel, H.; Zambelli, T. Force-controlled patch clamp of beating cardiac cells. Nano Lett. 2015, 15, 1743-1750.

[107] Saotome, K.; Murthy, S. E.; Kefauver, J. M.; Whitwam, T.; Patapoutian, A.; Ward, A. B. Structure of the mechanically activated ion channel Piezo1. Nature 2018, 554, 481-486.

[108] Nonomura, K.; Lukacs, V.; Sweet, D. T.; Goddard, L. M.; Kanie, A.; Whitwam, T.; Ranade, S. S.; Fujimori, T.; Kahn, M. L.; Patapoutian, A. Mechanically activated ion channel PIEZO1 is required for lymphatic valve formation. Proc. Natl. Acad. Sci. USA 2018, 115, 12817-12822.

[109] Cox, C. D.; Bavi, N.; Martinac, B. Biophysical principles of ion-channel-mediated mechanosensory transduction. Cell Rep. 2019, 29, 1-12.

[110] Li, M.; Dang, D.; Xi, N.; Wang, Y. C.; Liu, L. Q. Nanoscale imaging and force probing of biomolecular systems using atomic force microscopy: From single molecules to living cells. Nanoscale 2017, 9, 17643-17666.

[111] Müller, D. J.; Dufrêne, Y. F. Force nanoscopy of living cells. Curr. Biol. 2011, 21, R212-R216.

[112] Seifert, J.; Rheinlaender, J.; Novak, P.; Korchev, Y. E.; Schäffer, T. E. Comparison of atomic force microscopy and scanning ion conductance microscopy for live cell imaging. Langmuir 2015, 31, 6807-6813.

[113] Ando, T. High-speed atomic force microscopy and its future prospects. Biophys. Rev. 2018, 10, 285-292.

[114] Ossola, D.; Dorwling-Carter, L.; Dermutz, H.; Behr, P.; Vörös, J.; Zambelli, T. Simultaneous scanning ion conductance microscopy and atomic force microscopy with microchanneled cantilevers. Phys. Rev. Lett. 2015, 115, 238103.
[115] Yasui, M.; Hiroshima, M.; Kozuka, J.; Sako, Y.; Ueda, M. Automated single-molecule imaging in living cells. Nat. Commun. 2018, 9, 3061 .

[116] Sako, Y.; Minoghchi, S.; Yanagida, T. Single-molecule imaging of EGFR signalling on the surface of living cells. Nat. Cell Biol. 2000, 2, 168-172.

[117] Elf, J.; Li, G. W.; Xie, X. S. Probing transcription factor dynamics at the single-molecule level in a living cell. Science 2007, 316, 1191-1194.

[118] Tokunaga, M.; Imamoto, N.; Sakata-Sogawa, K. Highly inclined thin illumination enables clear single-molecule imaging in cells. Nat. Methods 2008, 5, 159-161.

[119] Kodera, N.; Yamamoto, D.; Ishikawa, R.; Ando, T. Video imaging of walking myosin $\mathrm{V}$ by high-speed atomic force microscopy. Nature 2010, 468, 72-76.

[120] Ying, Y. L.; Hu, Y. X.; Gao, R.; Yu, R. J.; Gu, Z.; Lee, L. P.; Long, Y. T. Asymmetric nanopore electrode-based amplification for electron transfer imaging in live cells. J. Am. Chem. Soc. 2018, 140, 5385-5392.

[121] Li, M.; Xi, N.; Wang, Y. C.; Liu, L. Q. In situ high-resolution AFM imaging and force probing of cell culture medium-forming nanogranular surfaces for cell growth. IEEE Trans. NanoBiosci. 2020, 19, 385-393.

[122] Li, L.; Guo, W.; Yan, Y. Z.; Lee, S.; Wang, T. Label-free superresolution imaging of adenoviruses by submerged microsphere optical nanoscopy. Light Sci. Appl. 2013, 2, e104.

[123] Lekka, M.; Pogoda, K.; Gostek, J.; Klymenko, O.; Prauzner-Bechcicki, S.; Wiltowska-Zuber, J.; Jaczewska, J.; Lekki, J.; Stachura, Z. Cancer cell recognition - mechanical phenotype. Micron 2012, 43, 1259-1266.

[124] Li, M.; Xi, N.; Wang, Y. C.; Liu, L. Q. Atomic force microscopy in probing tumor physics for nanomedicine. IEEE Trans. Nanotechnol. 2019, 18, 83-113.

[125] Tian, Y. M.; Li, J. H.; Cai, M. J.; Zhao, W. D.; Xu, H. J.; Liu, Y.; Wang, H. D. High resolution imaging of mitochondrial membranes by in situ atomic force microscopy. RSC Adv. 2013, 3, 708-712.

[126] Wang, X.; Liu, H. J.; Zhu, M.; Cao, C. H.; Xu, Z. S.; Tsatskis, Y.; Lau, K.; Kuok, C.; Filleter, T.; McNeill, H. et al. Mechanical stability of the cell nucleus-roles played by the cytoskeleton in nuclear deformation and strain recovery. J. Cell Sci. 2018, 131, jes209627.

[127] Stewart, J. B.; Chinnery, P. F. Extreme heterogeneity of human mitochondrial DNA from organelles to populations. Nat. Rev. Genet. 2021, 22, 106-118.

[128] Dan, K.; Veetil, A. T.; Chakraborty, K.; Krishnan, Y. DNA nanodevices map enzymatic activity in organelles. Nat. Nanotechnol. 2019, 14, 252-259. 